\title{
Real Time Autotuning for MapReduce on Hadoop/YARN
}

\author{
by \\ Maria Pospelova, B.Sc. \\ A thesis submitted to the \\ Faculty of Graduate and Postdoctoral Affairs \\ in partial fulfillment of the requirements for the degree of \\ Master of Computer Science (Accelerated) with Data Science \\ Specialization \\ Ottawa-Carleton Institute of Computer Science \\ Department of Computer Science \\ Carleton University \\ Ottawa, Ontario \\ July, 2015 \\ (C) Copyright \\ Maria Pospelova, 2015
}


The undersigned hereby recommends to the

Faculty of Graduate and Postdoctoral Affairs acceptance of the thesis

\title{
Real Time Autotuning for MapReduce on Hadoop/YARN
}

\author{
submitted by Maria Pospelova, B.Sc. \\ in partial fulfillment of the requirements for the degree of \\ Master of Computer Science (Accelerated) with Data Science \\ Specialization
}

Professor Herna L Viktor, Ph.D, External Examiner

Professor Michel Barbeau, Ph.D, Examiner

Professor Frank Dehne, Ph.D, Thesis Supervisor

Professor Anil Somayaji, Ph.D, Chair, Department of Computer Science

Ottawa-Carleton Institute of Computer Science

Department of Computer Science

Carleton University

July, 2015 


\section{Abstract}

Humanity entered the Big Data era passing through the zettabite mark at an exponential rate in just a few years, which made Big Data processing issues a reality for a wide range of business and academic applications. The Apache Hadoop is the most popular Big Data processing framework with the current market share of $\$ 3$ billion expected to increase to $\$ 50$ billion by the end of the decade; however, Hadoop deployment expansion does not proceed at the predicted rate.

One of the obstacles for further popularization of Hadoop is that performance is highly dependent on chosen settings. A nontrivial tuning process requires time and highly qualified human resources. Therefore, an automatic tuner is a desirable solution. Existent off-line tuning approaches require multiple repetitive executions of the job in order to find optimal tuning settings and, hence, are not applicable to use in most cases.

The presented work introduces a novel real time autotuning approach. The resource management parameters are tuned between execution waves by a modular autotuner connected to Hadoop architecture through YARN. The developed autotuner effectively intercepts a resource request, modifies it according to a tuning algorithm and passes it to YARN Scheduler. Such an approach not only carries high practical and theoretical value, but also opens a new horizon in the Hadoop/YARN automatic tuning development. 
This thesis is dedicated to my husband, Pino Guerra, and to my children, Matteo and Isabella. Thank you for your love, patience, and numerous sacrifices that you have made throughout my academic career. This thesis and the pursuit of my goals would not have been possible without your support. 


\section{Acknowledgments}

Foremost, I would like to express my special appreciation and my sincere gratitude to my advisor Professor Dr. Frank Dehne. You have been a tremendous mentor. I would like to thank you for encouraging my research and for allowing me to grow as a research scientist. Your advice on both research and on my career have been priceless.

I would also like to thank Professor Dr. Michel Barbeau, Professor Dr. Anil Somayaji, Professor Dr. Herna Viktor for serving as my committee members.

Furthermore I would like to thank my collaborator, Mikhail Genkin, for introducing me to the topic and sharing his invaluable experience and his talents. It would not have been possible without his crucial help.

My gratitude also goes out to the Parallel Computing and Data Science Research Lab members. I especially want to thank Dr. Sylvain Pitre and Andrew Schoenrock, who provided me with advice and support at times of critical need.

I take this opportunity to express gratitude to all of the School of Computer Science and the new Institute for Data Science faculty members for their help and support. 


\section{Table of Contents}

Abstract $\quad$ iii

Acknowledgments $\quad$ v

Table of Contents vi vi v

List of Tables $\quad$ X

List of Figures $\quad$ xi

1 Introduction 1

1.1 Data Science and Big Data Computing . . . . . . . . . . . . . 1

1.2 MapReduce ............................ 2

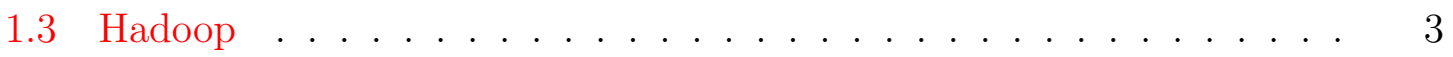

1.4 Auto-tuning Hadoop . . . . . . . . . . . . . . . . . 4

1.5 Thesis Motivation . . . . . . . . . . . . . . . . 5

1.6 Thesis Contributions . . . . . . . . . . . . . . 5

1.7 Thesis Organization . . . . . . . . . . . . . . . . 5

2 MapReduce 6

2.1 General Concept . . . . . . . . . . . . . . . . . . . . 6

2.2 MapReduce Implementations . . . . . . . . . . . . . . . . . . 6

2.3 Specifics of the Apache Hadoop . . . . . . . . . . . . . . . . 8

3 State of the Art $\quad 10$

4 Autotuning Algorithms 14

4.1 Simulated Annealing . . . . . . . . . . . . . . . . . . 14

4.2 Genetic Algorithm . . . . . . . . . . . . . . 16 
$4.3 \quad$ Hill Climbing . . . . . . . . . . . . . . . . . . . . . . . . 18

5 Hadoop Source Code $\quad 20$

5.1 Overall Architecture . . . . . . . . . . . . . . . . . 20

5.1.1 Original Apache Hadoop MapReduce . . . . . . . . . . . . . 21

5.1.2 Apache Hadoop Yet-Another-Resource-Negotiator (YARN) . . 22

5.2 Hadoop on YARN . . . . . . . . . . . . . . . . . . . . . . 23

5.2.1 YARN Application Execution Overview . . . . . . . . . 24

5.2.2 MapReduce Job Execution Overview . . . . . . . . . . . . . . 24

5.3 Detailed Exploration of the Current Implementation of the Most Relevant Packages . . . . . . . . . . . . . . . . 26

5.3 .1 Resource Manager . . . . . . . . . . . . . . . . . . 26

5.3.2 Scheduler Related Components . . . . . . . . . . . . . 27

5.3 .3 Client Interfacing Components . . . . . . . . . . . . 27

5.3.4 Connecting to the Nodes Components . . . . . . . . . 27

5.3.5 Application Masters' interacting components . . . . . . . . 28

5.4 Application Master . . . . . . . . . . . . . . . . . . . . . . . . . 29

5.5 YARN Schedulers . . . . . . . . . . . . . . . . 30

5.5.1 The FIFO Scheduler . . . . . . . . . . . . . . . 30

5.5.2 The Capacity Scheduler . . . . . . . . . . . . . . . . 31

5.5.3 The Fair Scheduler . . . . . . . . . . . . . . . . . . . 31

5.6 Tunable Hadoop Parameters . . . . . . . . . . . . . . . . . . . . 31

6 Autotuner Implementation $\quad 35$

6.1 Overview of the Autotuner Placement Within the Hadoop . . . . . 35

6.2 Autotuner Implementation Details . . . . . . . . . . . . . 37

6.2 .1 Autotuner Classes . . . . . . . . . . . . . . . . 37

6.2 .2 Autotuner Waves . . . . . . . . . . . . . . . . . 38

6.2.3 Execution order of the Autotuner . . . . . . . . . . . 39

6.2 .4 Explorer . . . . . . . . . . . . . . . . . . 40

7 Results $\quad 41$

7.1 Experimental Design . . . . . . . . . . . . . . . . . . . 41

7.1.1 Testing Cluster Configuration and Hardware . . . . . . . . . 41

7.1 .2 Hadoop Cluster Settings . . . . . . . . . . . . . . . . . 42 
7.1.3 Development environment set up with MiniYARNcluster on Eclipse development environment . . . . . . . . . . . . . . . 42

7.1.4 Benchmarks . . . . . . . . . . . . . . . . 44

7.1.5 TeraSort Benchmark . . . . . . . . . . . . . . 44

7.1.6 TPCx-HS Benchmark . . . . . . . . . . . . . . 46

7.1 .7 NMON . . . . . . . . . . . . . . . . . 47

7.2 Parameter Space Exploration _. . . . . . . . . . . . . 47

7.3 Default Baseline . . . . . . . . . . . . . . . . . . . 48

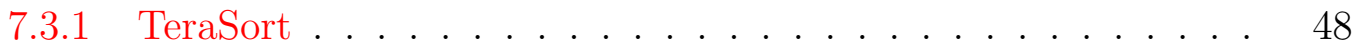

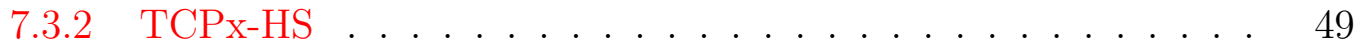

7.4 Preliminary Autotuner Results . . . . . . . . . . . . . . . 52

7.4.1 TeraSort ......................... 52

$7.4 .2 \mathrm{TPCx}-\mathrm{HS} \ldots \ldots \ldots \ldots \ldots$

7.5 Explorer . . . . . . . . . . . . . . . . 53

7.5.1 Explorer with 30-second Autotuner waves on memory and vCores 53

7.5.2 Explorer with 20-minute Autotuner waves on memory . . . . . 54

8 Discussion $\quad 57$

8.1 Innovation Concept. Real-Time Autotuning . . . . . . . . . . . . 57

8.1.1 Rational for real-time Hadoop YARN autotuning . . . . . . . 57

8.1.2 Autotuner Design Requirements . . . . . . . . . . . . . 58

8.2 Design Decisions Motivation . . . . . . . . . . . . 58

8.3 Result Evaluation . . . . . . . . . . . . . . . . . . . . . 62

8.3.1 Comparison of the Preliminary Autotuner Runs with Default and RoT Testings . . . . . . . . . . . . . . . . . 62

8.3.2 Explanation of the Results Obtained by Preliminary Autotuner Testing and Explorer Execution . . . . . . . . . . 62

8.3.3 Strength of the Current Autotuner Design . . . . . . . . . . 64

8.3.4 Weakness of the Current Autotuner Design and Ideas for Improvement ...................... 65

$\begin{array}{lll}9 & \text { Summary } & 67\end{array}$

9.1 Overview of the Results . . . . . . . . . . . . . . 67

9.2 Future Development . . . . . . . . . . . . . 68 


\section{List of Tables}

5.1 The Default and Rules of Thumb (RoT) settings for Hadoop Parameters 33

7.1 Completion Times for TeraSort on 100 GB Data with Different Values of mapreduce.* memory.mb Parameters with Calculated Standard Deviation ........................ 48

7.2 TPCx-HS Default Baseline Tests Results . . . . . . . . . . . . . . . 52

7.3 TeraSort on 100GB of data with Autotuner Empowered Hadoop with Hill Climbing Algorithm Tests Results . . . . . . . . . . . . . . . 52

7.4 TPCx-HS with Autotuner Empowered Hadoop with Hill Climbing Algorithm Tests Results . . . . . . . . . . . . . 53 


\section{List of Figures}

2.1 MapReduce Concept . . . . . . . . . . . . . . . . . . . . 7

5.1 Original Apache Hadoop MapReduce Architecture . . . . . . . . . . . 21

5.2 Apache Hadoop Yet-Another-Resource-Negotiator (YARN) Architecture 22

5.3 Apache Hadoop on YARN Structure . . . . . . . . . . . . . 24

5.4 MapReduce Jod Run on Apache Hadoop on YARN . . . . . . . . 26

6.1 Hadoop YARN Resource Requesting Scheme . . . . . . . . . . 36

6.2 Autotuner Placement in Hadoop YARN . . . . . . . . . . . 37

6.3 Autotuner Waves . . . . . . . . . . . . . . . . . . . . 39

7.1 TPCx-HS Execution Phases . . . . . . . . . . . . . . . 46

7.2 Graph of Completion Times for TeraSort on 100GB Data with Different Values of mapreduce. *. memory.mb Parameters . . . . . . . . . . . 49

7.3 NMON Analyzer results for 1TB Terasort test with default parameters. (A) Total CPU Utilization, (B) CPU Utilization per CPU, (C) Disk I/O, (D) Network I/O, (E) Network Packets. . . . . . . . . . . . 50

7.4 NMON Analyzer results for 1TB Terasort test with default parameters. (A) Total CPU Utilization, (B) CPU Utilization per CPU, (C) Disk I/O, (D) Network I/O, (E) Network Packets. . . . . . . . . . . . .

7.5 Hadoop empowered by the autotuner with Explorer algorithm with 30-second autotuner waves on memory and vCores . . . . . . . . . 54

7.6 Hadoop empowered by the autotuner with Explorer algorithm with with 20-minute autotuner waves on memory for the 1 TB TeraSort .

7.7 Hadoop empowered by the autotuner with Explorer algorithm with 20minute autotuner waves on memory for the $1 \mathrm{~TB}$ TeraSort (the later

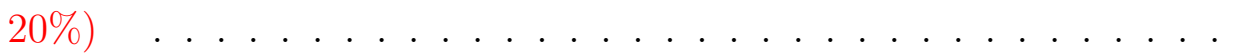

7.8 Hadoop empowered by the autotuner with Explorer algorithm with Explorer algorithm with 20-minute autotuner waves on memory for

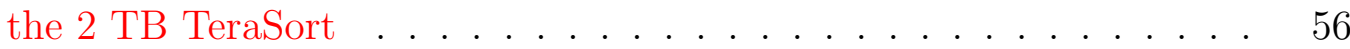


7.9 Hadoop empowered by the autotuner with Explorer algorithm with Explorer algorithm with 20-minute autotuner waves on memory for the 2 TB TeraSort (the later 20\%) $\ldots \ldots \ldots \ldots \ldots$

8.1 Comparison of the trend-lines: (A) Complete Terasort execution times, (B) Average container completion time per the autotuner wave during 1TB Terasort, (C) Average container completion time per the autotuner wave during 2 TB Terasort . . . . . . . . . . . . . 


\section{Chapter 1}

\section{Introduction}

\subsection{Data Science and Big Data Computing}

The estimated size of all digital data approaches 8 zettabytes, and this number is projected to continuously increase at an exponential rate. Hence, dealing with the Big Data processing problem is inescapable. Moreover, just a few years ago, this was a problem of the industry giants, such as Google and Amazon, now much greater share of business and academic projects must find a solution. The Big Data processing is a very new, extremely challenging and demanding area of research. Because of such circumstances, not only does a commonly accepted solution not exist, but also the definition of the term, Big Data, itself, is constantly challenged.

The Big Data term was added to the Oxford English Dictionary just two years ago [1], yet the term has been circulating in technical circles since the mid 90s. The most common definition describes Big Data as the high volume, high velocity, and high variety digital information collection. This definition is also known as "three V's" definition, and was originally given by Doug Laney in 2001 [2]. Yet many reputable experts disagree with this definition and propose their own. Nevertheless, most of the professionals agree that extremely challenging processing is one of the most prominent characteristics of Big Data.

In order to harvest useful information from the Big Data, the data science field emerged. Data scientists role requires domain knowledge, a mathematical and statistical base along with technical programming skills. However, most of all, it requires a curious, creative state of mind, which prompts data scientists to explore the data in many different ways: testing out various hypotheses in a search of interesting patterns and correlations, and translating solutions from one domain to another. For instance, 
Davenport and Patil brought up an example of a data scientist who not only found similarity between a DNA sequencing problem and a fraud investigation they were working on, but also successfully applied an existing solution to the new domain [3]. Moreover, the experimental nature of data science requires a quick response to a wide variety of data processing tasks.

\section{$1.2 \quad$ MapReduce}

Before the popularization by Dean and colleagues, in 2004, [4], as a panacea for all Big Data problems, the MapReduce technique was already known among distributed and parallel computing professionals. However, Google's publication certainly brought the technique to light for the rest by adding a new spin and causing its popularity to spread like wildfire.

MapReduce is a computational model based on functional programming concepts of list processing. One of the key concepts of functional programming, data immutability, supports reliable and efficient distributed data processing without costly and fault-prone current state synchronization issues. Each task does not modify input key-value pairs, but produces new ones with modified values, which are forwarded by the Hadoop system into the next phase of execution. A MapReduce program is composed of two list-processing idioms, which are exposed to the user: map, and reduce. These terms are also not new and had been previously used in other list-processing languages such as LISP, Scheme, and ML.

The first phase, mapping, executes Mapper, a function, which transforms the elements of the input list to output data elements, acting on each element individually. Next, the shuffling phase, which is transparent to the user, rearranges output produced by Mapper. Shuffle process results in all data pairs with the same key being localized together and assigned to the same reducer. Then, in the reduce phase, Reducer, a function which aggregate values together, receives an iterator for the produced list and combines these values together, returning an output value.

Since its introduction, MapReduce gained a strong position for Big Data processing applications of various natures in both industry and academia. The most popular MapReduce implementation is the Apache Hadoop. 


\subsection{Hadoop}

Initiated by Doug Cutting and Mike Cafarella, an open-source search engine, Nutch, was inspired by two famous Google papers [5,6]. Later in 2006, when Cutting started his work at Yahoo, Hadoop was given its name. Moreover, the original Hadoop running cluster, which was built as proof of a concept for a new search engine, was noticed by Yahoo's data scientists for its surprisingly good performance. The success of Yahoo's search relevance or Yahoo's advertising revenue analytics built on Hadoop led to the "behind every click" keystone position at Yahoo. Together, Yahoo developers and open-source contributors brought Hadoop to the solid state, which allowed it to be chosen as a primary MapReduce education framework. A cluster of 2000 Hadoop nodes was sponsored by IBM, Google and the National Science Foundation. Later, Cloudera and Hortonworks, commercial Hadoop companies, played an important role in increasing the popularity and furthering the business deployment of the Hadoop.

Some of the advantages of an open-source project is its ability to react quickly to new industry demands, and incorporate innovative technologies. The evolution of Hadoop, since its creation, is tremendous. Nevertheless, the recent transition to YARN-based architecture could be considered the most significant framework upgrade since Hadoop's creation. YARN (Yet Another Resource Negotiator) expanded the Hadoop programming model from strictly MapReduce to a variety of processing engines. YARN architecture for resource management provides more consistent performance, better governance of the data across Hadoop clusters, and improved security.

The new resource management system built upon the container concept. Containers are resource partition abstractions of the specified size, which are used as a currency to negotiate the distribution of the Hadoop cluster resources among the applications. The Application Master requests a specific number of containers with a certain size of the memory and a number of virtual cores. The Resource Manager collects the requests and passes them to a YARN scheduler. The scheduler distributes currently available resources by partially satisfying incoming requests, as total of requested resources is usually much greater than the total of currently available resources. This action is repeated periodically, on each heartbeat. Simultaneously allocated containers form a wave. Considering allocation happens on almost every heartbeat, by default, the heartbeats emitted every minute number of waves per application is significant. The size of memory and the number of virtual cores 
per container are set in configuration file by the user, and are static throughout the job execution. Moreover, they affect job completion time significantly.

Since its creation, Hadoop has come a long way. In 2011, it was proclaimed the most important innovation, surpassing Apple's iPad, and receiving the top prize of the MediaGuardian Innovation Award. The future is also bright; IDC predicts that Hadoop-MapReduce ecosystem will be worth $\$ 813$ million by the next year [7]. Furthermore, the experts suggest that its actual economical impact will be significantly greater.

\subsection{Auto-tuning Hadoop}

Tuning a Hadoop MapReduce job is a nontrivial task. Hadoop exposes over two hundred parameters, many of which have a severe impact on job performance. A poorly configured job can result in extremely slow execution times or even failure. Currently, the industry standard is manual tuning, which requires iterative repetition of the job execution with adjustment of xml configuration files by highly qualified professionals. Such professionals are in high demand. Moreover, as the range of Hadoop users expands, many of the users cannot obtain such employees due to a shortage in the availability or resources.

The growing importance of Apache Hadoop, the severity of the impacts of parameter selection, along with the limitation of resources, has led to vivid interest in the research community. A number of studies explored the variety of off-line tuning. Some of them were based on a statistical model with the incorporation of "rule of thumb" knowledge, such as the Starfish project $[8,9]$. Another group of projects favoured an algorithmic approach such as the Gunther [10] and Panda [11] projects. Many of them achieved impressive results in the improvement of job completion time. Unfortunately, such results could be translated into a practical value only for use cases in which the same job is repetitively executed on the same size data, as any changes in the job or the size of data will require adjustments in tunable parameters. Therefore, for many data science use cases, repetitive iteration of the single job by off-line autotuner not only does not speed up the process but also makes job completion time magnitudes slower. 


\subsection{Thesis Motivation}

The answer to the stated problem is a real time Hadoop autotuning process, which allows the optimization of cluster deployment and completion time of a MapReduce job executed on Hadoop/YARN during a single run. A real time autotuning solution will extend to all Hadoop use cases including repetitively and single time executed MapReduce jobs. Moreover, a real time autotuning would solve the problem with human recourse shortage, opening effective Hadoop/YARN deployment to a much wider sector of users.

\subsection{Thesis Contributions}

The work presented in the thesis lays a strong base for the complete real time autotuning Hadoop/YARN solution and provides proof of a concept that such tuning is possible through a working autotuner implementation. The presented autotuner adjusts container parameters (size of memory and number of virtual cores) in real time, during a single job execution. Moreover, an effective cost evaluating function, which estimates total job completion time based on information collected in a fraction of a total job, was developed. Furthermore, the test results confirmed the effectiveness of the cost function, along with the autotuner architecture and its Hadoop/YARN integration scheme. Hence, the thesis work lays a solid foundation for the development of an effective real time Hadoop/YARN autotuning solution.

\subsection{Thesis Organization}

Chapter 2 introduces the main concepts of MapReduce and its implementations. Chapter 3 summarizes state of the art of autotuning research for Hadoop MapReduce jobs. Chapter 4 briefly introduces the commonly used automatic tuning algorithms for Hadoop, which were considered for the current autotuner. Chapter 5 reflects the main points and concepts of Apache Hadoop Architecture based on a detailed study of Hadoop source code and documentation. Chapter 6 describes the developed real time autotuner implementation. Chapter 7 describes experimental design and collected results. Chapter 8 presents the analysis of the observed results. Chapter 9 summarizes findings and outlines prospective steps for future development. 


\section{Chapter 2}

\section{MapReduce}

\subsection{General Concept}

In 2004, Dean and Ghemawat introduced the MapReduce programming concept as an answer to the ever growing demand for fast and fault-tolerant Big Data processing [5]. The authors aimed to create a simple yet effective programming model which allows the programmer to focus on the computation task at hand abstracted from the complications of a distributed system by such qualities as fault tolerance, load balancing, data distribution and task parallelization. Concisely, MapReduce applications consist of map and reduce functions. First, all data is distributed among processing units, nodes. Then, each assigned node executes a map function, which creates key-value pairs from the data it has locally on the node. Next, the shuffle stage, moves key-value pairs in such a way that all data units with the same keys are located on the same node. Lastly, the reduce stage starts, and one or more reducers compute the final output. The overall scheme of a process is shown in Figure 2.1. Note that distribution of the files among the nodes, and shuffling between map and reduce stages is not exposed to the application developer and is facilitated by a MapReduce framework.

\subsection{MapReduce Implementations}

Currently, there are a number of various MapReduce implementations including Hadoop, Spark, Disco, MapReduce MPI, Phoenix, MARIANNE, and MARISSA. In addition to the open source and academic projects named above, a number of 


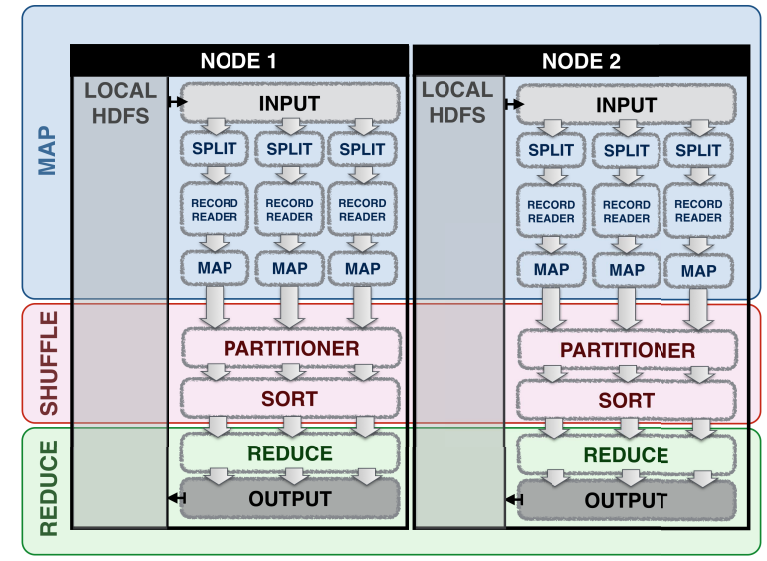

Figure 2.1: MapReduce Concept

proprietary internal MapReduce implementations have been developed for internal use.

Hadoop is the most popular implementation with exponential growth of its popularity. This implementation is produced and distributed under an Apache open source license [12]. Most of the implementation is written in JAVA, but a user can select another language to develop MapReduce applications.

Spark is another MapReduce implementation strongly gaining popularity in recent years [13]. Originally Spark was developed by the AMPLab at UC Berkeley, currently moved to an Apache Incubator project. Spark is designed for performance and belongs to "next generation" MapReduce implementations. Spark provides APIs for Scala, Java, and Python. In addition, it provides additional libraries such as Shark for SQL type querying, MLlib for machine learning applications and streaming API.

Disco was originated at Nokia and later became an open project [14]. The implementation is written in Python and Erlang. Nokia used Disco in production for several years. Disco consists of a relatively small code base, which provides a robust and effective data analytics tool.

MapReduce MPI is authored by Steve Plimpton and provides bindings for $\mathrm{C}++$, C, and Python [15]. MapReduce MPI is a library, which implements MapReduce for distributed-memory parallel machines on top of the standard MPI message passing scheme. MapReduce MPI is distributed freely under the terms of the modified Berkeley Software Distribution (BSD) License.

MARIANNE, developed by Dede [16], is an academic project and provide MapReduce framework for parallel networked file systems (GPFS). 
Phoenix was originated in Stanford University and written in $\mathrm{C}++$. The original publication was produced in 2009 by Yoo and colleagues [17]. The Phoenix MapReduce framework operates at the thread level. Apache Phoenix converts SQL query into a series of HBase scans and then manages their execution in order to produce regular JDBC result sets.

\subsection{Specifics of the Apache Hadoop}

The Apache Hadoop software library is an open-source software framework for reliable, scalable, distributed computing. It is designed for processing large data across multiple computer nodes. Originally, Hadoop was based exclusively on the Map Reduce programming model, but recently it expanded to support additional programming models. The projects composed of multiple modules, including Hadoop Common, Hadoop Distributed File System, Hadoop YARN and Hadoop MapReduce.

Hadoop Common is a module composed of common utilities and libraries responsible for the support the other Hadoop modules. Hadoop Common assumes hardware failures and must be handled by the software.

Hadoop Distributed File System (HDFS) is a data scalable, fault-tolerant, costefficient storage module that provides high-throughput access to application data. HDFS is a Java-based file system designed to span large clusters. HDFS offers a number of valuable features such as rack awareness, minimal data motion, utilities, rollback, standby NameNode, and operability.

Hadoop YARN (Yet Another Recourse Negotiator) is the most recent addition to Hadoop architecture. The YARN module controls job scheduling and cluster resource management. YARN could be viewed as the architectural center of Hadoop. The YARN based architecture allowed Hadoop to evolve from being MapReduce based to a more general large data processing platform. Together, HDFS and YARN form the data management layer of the Apache Hadoop framework.

Currently, the Hadoop MapReduce module is a YARN-based large data processing system based on MapReduce programming paradigm. The module provides machinery to process Java and non-Java user applications written in MapReduce style functional programming constructs for processing lists of data. MapReduce and HDFS components were originally derived from Google's MapReduce [5] and Google File 
System (GFS) [6] papers, respectively. Original Apache Hadoop Map Reduce implementation had a number of limitations. For instance, the number of map and reduce slots was predetermined for each of the task category at the beginning of the execution and stayed static throughout the job. Therefore, if one of the tasks takes an excessive amount of time, then the entire MapReduce job could be delayed. MapReduce 2.0 (MRv2), based on YARN architecture, has more effective resource management system which partially overcomes limitations of the first version.

In addition to these key components, a number of Hadoop-related projects spawn at Apache Hadoop, including Ambari, Avro, Cassandra, Chukwa, HBase, Hive, Mahout, Pig, Spark, Tez, ZooKeeper. Each of the projects extend Hadoop functionality or modify it in order to achieve optimal performance for a specific set of tasks.

For example, Cassandra is a highly scalable high-performance database with proven fault-tolerance [18]. Cassandra's Hadoop implements the same API as HDFS, in order to achieve input data locality. Therefore, Cassandra is deployed seamlessly with Hadoop. Cassandra is deployed by over 2500 companies, including Apple, Netflix, and Easou. The largest installations are composed of more than 75,000 nodes and store over $10 \mathrm{~PB}$ of data.

Spark is a fast, general engine for large data distributed processing [13,19]. Spark is compatible with Hadoop; hence, it could be deployed with Hadoop on top of the Hadoop YARN, or as a standalone framework. Spark exhibits high performance by operating on data reliably stored in memory, hence avoiding costly disk accesses. Spark operation can be based on various data stores such as HDFS, Cassandra, HBase, and S3. 


\section{Chapter 3}

\section{State of the Art}

The performance of Hadoop jobs is sensitive to underlying hardware, network infrastructure, JVM configuration and the Hadoop parameter settings. All of these challenges had been addressed by multiple research groups, revealing the importance of each of the levels $[9,10,20,21]$. While the first three levels could be addressed once when a cluster is composed, the last must be attended each time when a new MapReduce job is executed. Apache Hadoop exposes over 200 tunable parameters, $10 \%$ of which have a great impact on performance and effectiveness of the execution $[8,10,22]$. Exist so called Rule of Thumb (RoT) that are based on parameter tuning guides released by manufacturers [23-25]. The difference in processing speed between commonly used RoT settings and optimal configuration could be up to 100 fold $[10,26]$.

In general, autotuning approaches can be divided in two categories: deterministic and non-deterministic (heuristic). The first category relies on the meticulous exploration of the search space, covering every possible solution. The method gives you a guarantee than the found solution is the optimal one. Such a guarantee comes at the cost of the long and extensive computation time and, in some cases, simply is not feasible. The second category explores parts of the search space in such a way that resulting values are most likely to be an optimal solution, but are not a guarantee. The advantage of this method reduced the amount of computation required. Examples of deterministic solutions include A-tune, Manimal, Panacea, HAT and Starfish [8, 9, 20, 21, 27, 28].

The second category contains solutions based on heuristic approaches such as Gunther, and machine-learning framework authored by Yigitbashi and colleagues $[10,22,29]$. Hadoop autotuning was explored by multiple research groups. 
A language approach was explored, in 2009, by Schaefer and colleagues [20], who developed an autotuning language for Map Reduce Atune-IL, which allows a user to explore a selected set of tunable variables, such as the of threads or numbers of map and reduce jobs, within defined by user limits. Atune-IL also allowed a user to explore possible alternatives of the algorithmically blocks of the code. This approach automates manual tuning to some extent, reduces search space and translates the solution search to a simpler language tool; however, it requires a user to have the expertise of Hadoop tuning in order to select important for the MR job tunables, to set up limits and the size of the step for each tunable. Moreover, Atune-IL requires a developer learn new syntax, specific to the language.

In 2010, one of the Hadoop creators, Michael Cafarella, together with Christopher Re, introduced the wholly-automatic optimization framework, Manimal [21], which uses static code analysis to detect MapReduce program semantics. The authors combine widely practiced RDB tuning techniques with MR model resulting in $63 \%$ performance gain on selection tuning. The research suggests that selection tuning success could be expanded on many other code structures such as projections, and data compression.

Another way of approaching an autotuning problem is incorporating tuning algorithms on the compiler level. An autotuning compiler for MapReduce, Panacea, was proposed in 2012 by Liu and colleagues [27]. Panacea is able to optimize parameters and MR-blocking with little or no input from the developer. Panacea claimed up to three-fold performance improvement without user input. A fixed subset of tunable parameters was explored within limits, set by a user or auto-determined by Panacea. Parameters varied independently in a process of autotuning, which authors admit is a suboptimal solution due to specifics of the search space landscape.

A history-based auto-tuning framework for MapReduce, HAT, was introduced by Chen and colleagues in 2013 [28]. HAT tunes the weight of each stage of a map and a reduce task according to value of the tasks in the history and orchestrates Hadoop back up task execution according to the current and historical weights of the tasks, resulting in $37 \%$ improvement of execution time.

Starfish is a cost-based Hadoop autotuning framework developed at Duke University by Babu and colleagues [8,9]. Starfish takes into account different levels of MapReduce program, adjusting various decision points, which include provisioning, 
optimization, scheduling, and data layout. The heart of the framework is a Whatif engine, which employs a combination of simulation and model-based estimation. The authors succeeded in applying a popular in relational database tuning cost-base towards the MapReduce concept.

In 2013, Liao and colleagues analyzed model-based approaches for Hadoop MapReduce optimization and identified major limitations [10]. The research group proposed and implemented, Gunther, a search-based auto-tuner. The comparison of several global search algorithms leads to the selection of GA as a search strategy. The modified GA was evaluated on two clusters. Experimental results demonstrated that Gunther achieves near-optimal performance in a small number of trials $(<30)$ and yields better performance improvement than rule-of-thumb settings and a cost-based auto-tuner.

As another example of the heuristic approach published in the same year as Gunther, is research conducted by Bezhad and colleagues. They also demonstrated that a GA -based parameter search combined with dynamically intercepted HDF5 calls can result in up to 100 fold I/O write speedup for some tasks [26].

Another niche of heuristic algorithms, machine learning, was explored by Yigitbashi and colleagues [22], who analyzed various machine learning-based performance models. The analysis was conducted on two standard Hadoop benchmarks: sort and word count, with datasets of various sizes and led to a conclusion that Support Vector Regression exhibits the best performance between machine learning methods. The Support Vector Regression based autotuner also outperforms a well-known, cost-based auto-tuner, Starfish.

Another search-based evolutionary computation approach was conducted by Filho and colleagues, in 2014 [29]. They propose an adaptive tuning mechanism that enables the setting of specific resources to each job within a query plan. A data structure mapping a job to tuning solutions is created based on an analysis of the source code and $\log$ files.

Notice that all presented solutions are developed based on the off-line autotuning concept. Therefore, these solutions require multiple executions of the full job in order to produce optimized tuning values. During the work on the current project a research introducing mrOnline was published by Li and colleagues [30]. mrOnline is an online autotuner for the YARN framework, which was evaluated on a 19-node cluster and claims up to $30 \%$ performance improvement compared to the default settings. 
mrOnline built on top of HDFS and the cost evaluation is done via autotuner daemons running in each of the name nodes. Moreover, the autotuning engine is powered upon a pre-programmed set of rules. Unfortunately, mrOnline was built in collaboration with IBM research; hence, details of implementation and the source code were not made available to the public.

The presented selection of autotuning investigations show that Hadoop autotuning is, indeed, a very active research field. There is multiple approaches tested under different circumstances. A comparison between such solutions, or even directions of autotuning approach, is extremely difficult due to specifics of domain and overall in the maturity of Hadoop, and MapReduce, in general. It is clear that a pure modeling and cost-based approach does not reach a desirable level of improvement and, in some cases, its flaws are especially visible [8,10]. Yet, heuristic approaches, despite the achievement of superior results, require multiple iterations, which, considering the size of computation for an average MR job, make real life employment of such concepts unfeasible [22]. Nevertheless, all the publications, excluding mrOnline, persuade the off-line autotuning approach, which limits application for the final product. The emergence of on-line tuning research, mrOnline, confirms that real time tuning is a highly desirable, yet underexplored direction, of Hadoop/YARN automatic tuning. 


\section{Chapter 4}

\section{Autotuning Algorithms}

A number of the approaches were applied to autotuning in general and, in particular, to the Hadoop autotuning. In this chapter, the most popular and effective algorithms will be presented.

\subsection{Simulated Annealing}

Simulated annealing is an extremely popular local search algorithm. The popularity of simulated annealing roots in the simplicity of the concept, the low computational requirements and relatively good performance. The concept was derived from the physical process of the annealing solids. In metallurgy, annealing raises its temperature above re-crystallization mark, followed by cooling below it. It is extremely important that the cooling process is conveyed in a slow, careful manner. The desired state of the solid is obtained only if heating was sufficiently high and cooling was sufficiently slow, allowing particles to arrange in a highly structured grid, which corresponds to the lowest energy level. The cycle could be repeated numerous times.

First, the concept of annealing was translated to computer science in 1983 by Kirkpatrick [31] and independently by Cerny in 1985 [32]. Translation from physical to computer science optimization problem is achieved by the assumption of the two following equivalences. First, solution vectors in an optimization problem are equivalent to states of the physical system. Second, the cost of the solution, determined by the cost function, is equivalent to the energy of a physical state. Moreover, the temperature role is played by the control parameter. Originally, the simulated annealing algorithm was very close to the physical model; later in its development, it deviated from it to the higher abstraction level. 
Let us view the parameter optimization as a minimization problem. Note this easily translated into a maximization problem; hence, it could be used without loss of generality. The algorithm probabilistically re-samples the problem space. The acceptance of new samples is based on the cost and probabilistic components. Over the execution time of the algorithm, "temperature" or control criteria $\left(c_{k}\right)$ decreases from relatively large values to almost zero. If the cost of a new parameter is greater or equal to the current cost, it is accepted and becomes a new current (see Algorithm 1: lines 6-7). Otherwise, the probability of acceptance of a new value depends on value of $\exp ((f(i) f(j)) / c)$ through comparison of that value to a random number between 0 and 1 (see Algorithm 1: line 8). After each iteration, the new length and new control value are calculated (see Algorithm 1: lines 12-13). Other steps of the algorithm are routine. It starts from the initialization of the starting state, which consists of a current parameter $\left(i_{s}\right.$ tart $)$, initial control parameter $\left(c_{0}\right)$ and initial length $\left(L_{0}\right)$. Length value determines how many samples will be taken at the particular iteration. Iterations are repeated until a stopping condition is met (see Algorithm 1: line 3). Each iteration has a number of samples generated (see Algorithm 1: line 5) and checked against current value. The number of samples is determined by the length value (see Algorithm 1: line 4).

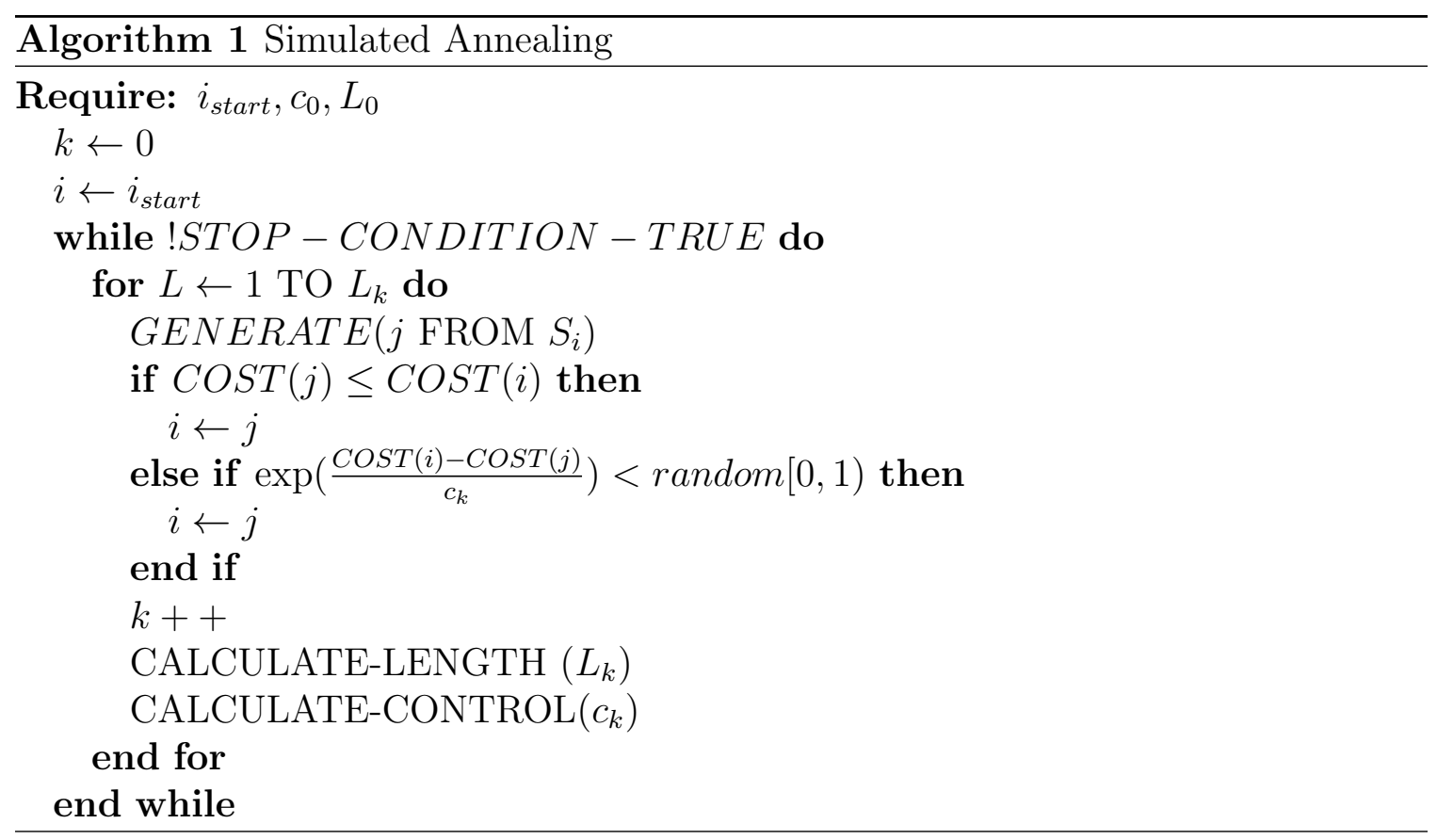


Since its introduction, simulated annealing had been successfully applied to numerous problems in a wide variety of research areas including molecular physics, code processing, VLSI design, operations search and autotuning. An example of an autotuning application of simulated annealing is the Panda project [11] studied automatic performance optimization for collective I/O.

The good performance, simplicity of implementation, applicability to a wide range of the problems and flexibility resulted in the popularization of simulated annealing algorithm. Nevertheless, the application of simulated annealing to concrete problems requires tuning of itself. In particular, finding an optimal cooling schedule and localizing domain neighborhoods for it could lead to extensive experimental overload.

\subsection{Genetic Algorithm}

A genetic algorithm is based on a process of natural selection. First, this idea arose in Turing's publication on computer intelligence, in 1950 [33]. Four years later, a publication with the first implementation of the genetic algorithm was produced by Norwegian-Italian mathematician, Nils Aall Barricelli [34]. Classical implementation of the genetic algorithm closely mimics biological evolution. The algorithm proceeds by repeated modification of a population of individual solutions. During each cycle, the genetic algorithm uses one of the selection mechanisms to choose individuals who will become parents for the individuals of the next generation. Selection mechanisms includes the random component and fitness component, which directs the overall progress of the population toward an optimal solution.

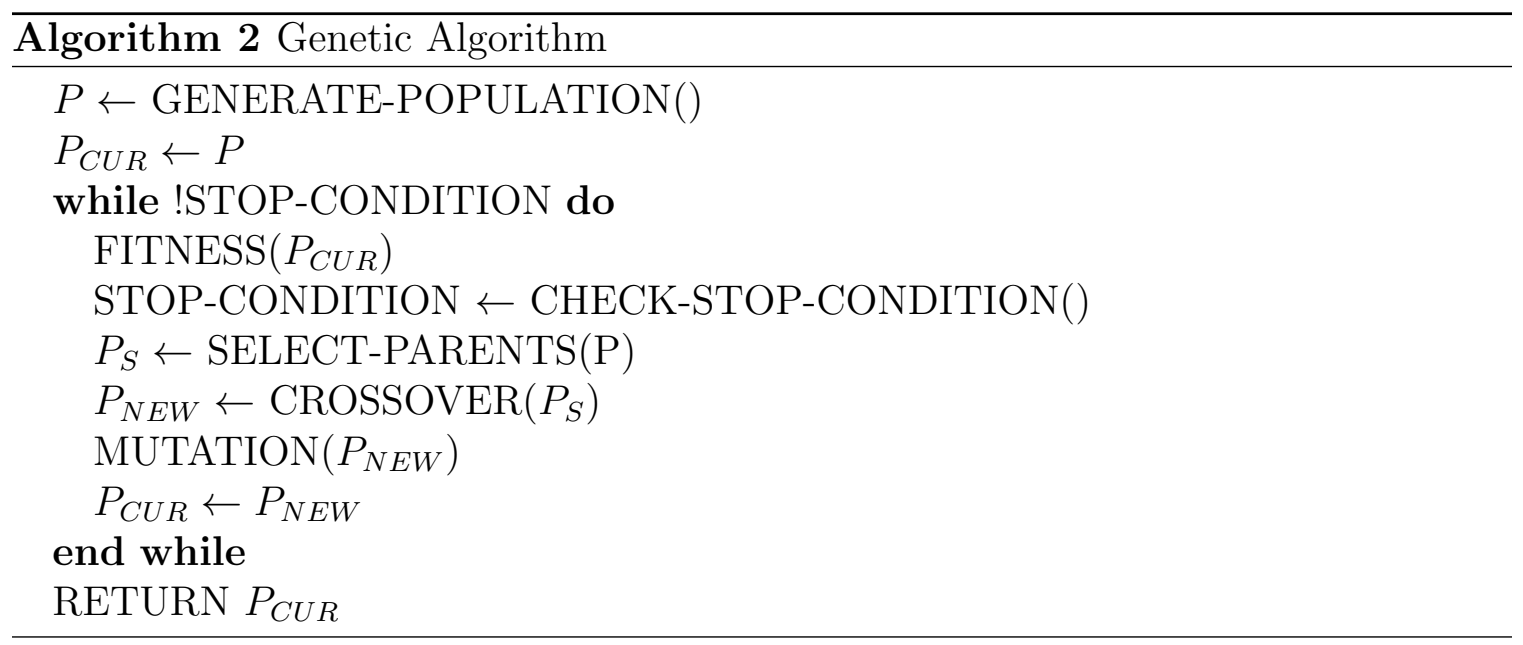


The typical genetic algorithm consists of the five main steps: generation of the original population; evaluation of the each of the individual with a fitness function; selection of the parents according to a selection function; production of the next generation with optional employment of crossover and mutation functions; check stopping conditions and, if they are satisfied, return the best solution, so far. Commonly used stopping conditions are composed of optimal, or near to optimal, solutions and generation number maximum. The last four steps are repeated until the stopping condition is met.

Strong points of the genetic algorithm approach include the ability to reach a near optimal solution and the algorithms flexibility and suitability for complex problems with multiple objectives. Moreover, the effective selection of the population size, selection, crossover and mutation methods provides an effective mechanism, which avoids local minimal in non-unimodal complex search surface. The downsides of a genetic algorithm methodology include multiple repetitions, which could be unsuitable for real world problems. For example, if a population consists of 12 solutions, which is an extremely small population, and 30 generations are required to reach a near optimal solution, then 360 evaluations are required, in total. As these evaluations run sequentially, for a 5-minute job, it will take 30 hours of tuning to discover the best solution and, for 1-hour job, it will take 15 days of continuous computation. The decrease in population size or number of generations has a negative effect on the results. Another problem with the genetic algorithm approach is that the approach is extremely sensitive to the selection of its own parameters, such as choice of the fitness, selection, mutation and crossover methods, along with the previously mentioned size of population and maximal number of generations parameters. Hence, tuning of the autotuning algorithm becomes an additional task.

The practical application of genetic algorithms spreads widely across many fields including various optimizations and automatic tuning problems in engineering, computational science, bioinformatics, signal and image processing, risk analysis, robotics, economics, molecular chemistry, computational physics and many others. During research of the existent work of MapReduce and Hadoop automatic tuning, a number of solutions employed genetic algorithm. For instance, the Gunther project developed by Liao and colleagues explores parameter space by means of a genetic algorithm and achieves near-optimal performance [10]. Another example of an auto-tuning system, which employs genetic algorithm for autotuning is a parameter optimization system 
for I/O performance of HDF5 application developed by Bezhad and colleagues [35]. This system achieves impressive speedups up to fifty times faster than the baseline.

\subsection{Hill Climbing}

Hill climbing, also known as greedy local search, is a popular gradient-based heuristic algorithm often used for optimization problems. The name of the algorithm comes from its analogy with the climbing of a hill. First the algorithm is starting at the base with a sub optimal solution, which needs improvement. Than continue to improve it with small steps in direction of the top of the hill, the optimal solution.

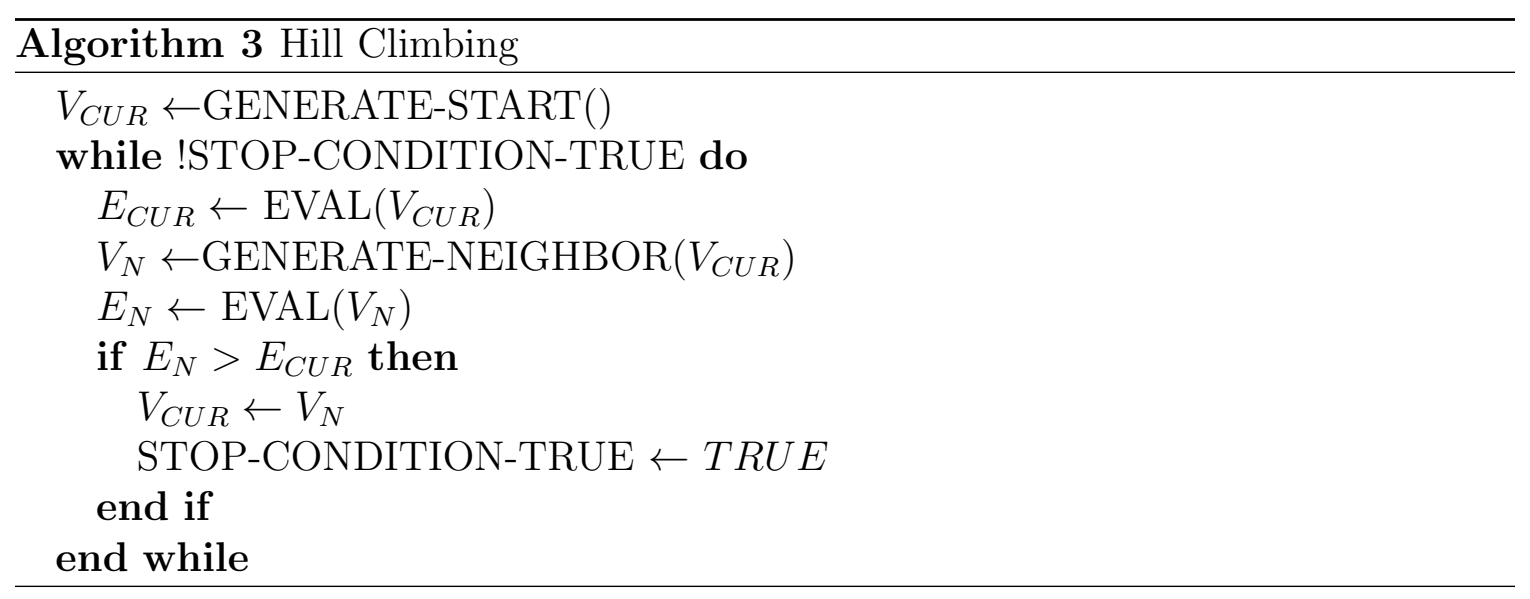

The hill climbing algorithm starts at a given point in the search space, evaluates it, then produces one or more neighbours of that point, then also evaluates these solutions with specific fitness function. Next, current point fitness is compared to the fitnesses of the neighbours and the best is selected to be the next current point. The cycle is repeated until stopping conditions are met. Stopping conditions could take effect when the maximum number of iterations is reached or the best point of the neighbourhood is found. In other words, no local move would improve the solution. That approach makes hill climbing to be extremely sensitive to producing suboptimal solutions by "getting stuck" at a local optimal solution. One of the solutions for this problem is to allow the algorithm to proceed to equal or worse than current solutions, keeping track of the current best solution. In this case, the stopping condition could have a different set of rules. This relaxed approach is a key component of local search methods for the Boolean specifiability (SAT) problems, such as GSAT and 
Walksat [36]. Another way to escape local optimal solutions is to restart the climb from a previously visited point with an unexplored neighbor or have multiple climbers exploring the search space simultaneously. These are stochastic variations of a hill climbing algorithm.

A great example of an effective variation of the hill climbing algorithm is an awardwinning Late Acceptance Hill Climbing algorithm [37]. The main idea of the Late Acceptance Hill Climbing algorithm is that it accepts a candidate solution if it is not worse than the solution, which was "current" several steps before. It is impressive that such a simple concept outperformed many complex machine learning approaches.

Therefore, modified hill climbing algorithms produce surprisingly good results, despite their simplicity and surpass the performance of many other much more complex heuristics. Moreover, most of the optimizations do not require as large a number of executions as some other methods, such as genetic algorithms do. Another advantage of the hill climbing technique is that it does not depend on the quality of a coolingschedule, selection, crossover, and mutation methods, size of population and other parameters that greatly affect previously discussed algorithms. 


\section{Chapter 5}

\section{Hadoop Source Code}

\subsection{Overall Architecture}

Most Hadoop source code is written in Java. Critical code is written in C, for performance reasons. Hadoop 2.6.0 can be run on various Linux distributions and Windows. The original Hadoop framework was composed of two layers: MapReduce and HDFS. The MapReduce layer is the computational layer. HDFS is the layer responsible for file storage and organization. Both layers are based on master/slave node architecture. Such architecture simplifies design by providing the global knowledge base, which includes job scheduling and block placement information. The disadvantage of this architecture is that the master node becomes a single point of failure. In some cluster configurations, the situation could be improved by a hot standby for the master node. Nevertheless, Hadoop clusters are extremely robust against slave node failures. Slave node data is duplicated and could be reconstructed by remaining slave nodes in the event of failure. While in the traditional distributed computing model, data is moved to the place of computation; in MapReduce the computation is moved to the data. Therefore, high data throughput with minimal data movement, so desirable for Big Data processing, is achieved. Since Apache Hadoop 2.2.0, there are two implementations of the MapReduce programming model available: the original Apache Hadoop MapReduce model, and the new Apache Hadoop Yet-Another-Resource-Negotiator (YARN) model (Figures 5.1, 5.2). 


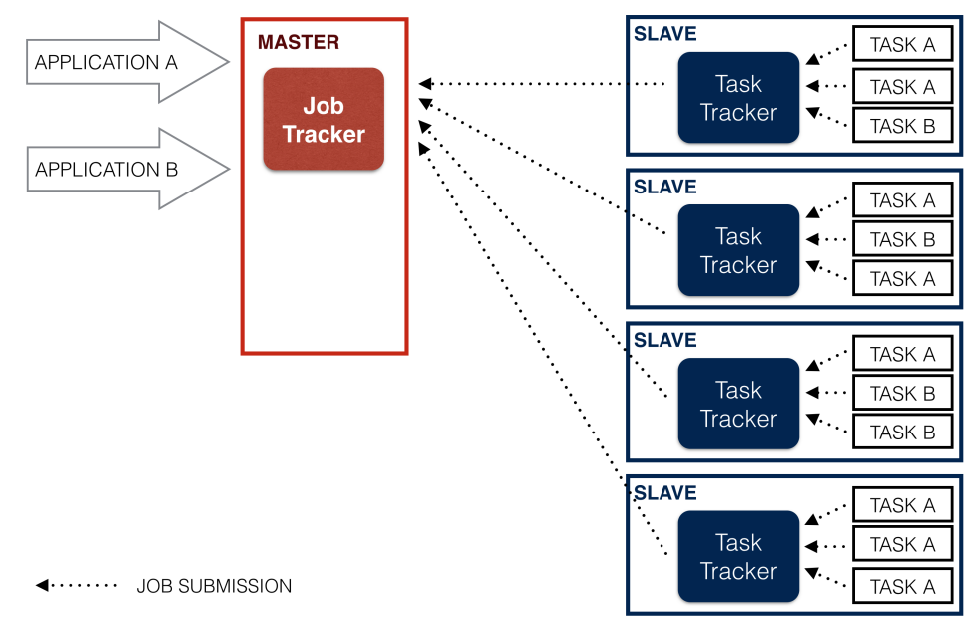

Figure 5.1: Original Apache Hadoop MapReduce Architecture

\subsubsection{Original Apache Hadoop MapReduce}

The Original Apache Hadoop MapReduce consists of one JobTracker, the master component, and multiple TaskTrackers, slave components, each of which assigned to an individual slave node. The JobTracker is a service that distributes MapReduce tasks to the individual nodes. It assigns tasks to the nodes with the data or at least on the same rack with the data. The JobTracker monitors completion of the tasks, records failures and controls the number of attempts. The TaskTracker monitors tasks running on the single node and reports to the JobTracker. A TaskTracker is limited by the number of map and reduce job slots of the node. If all slots are occupied, no more tasks can be executed on the node. Hence, if all map slots are occupied and reduce slots are free, additional map jobs cannot be initiated using a reduce slot. Therefore, the node is underutilized. In order to solve this issue, a new MapReduce implementation, which addresses resource management weaknesses, was proposed. 


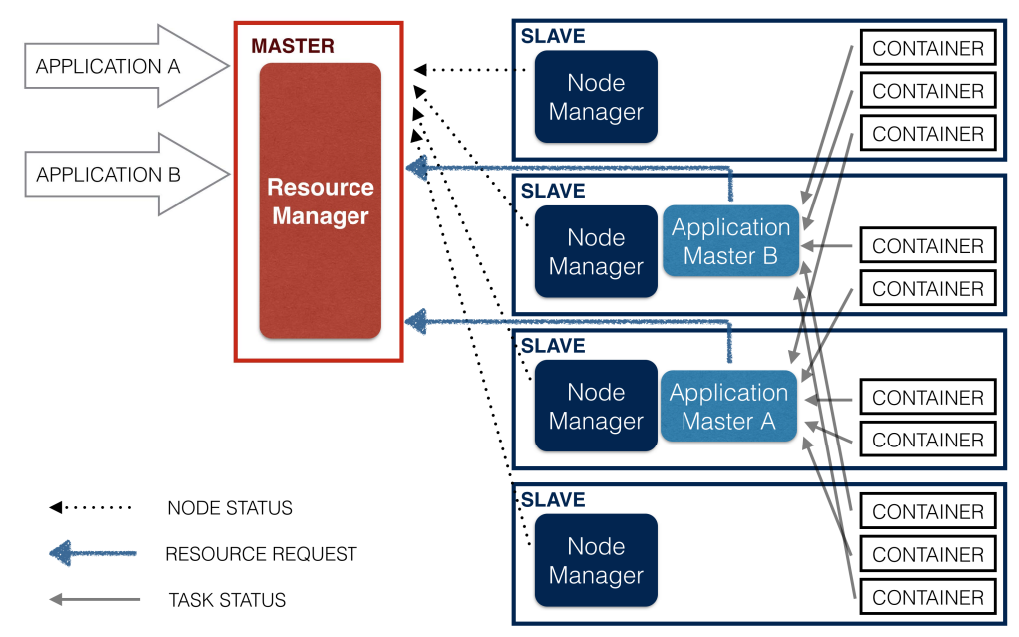

Figure 5.2: Apache Hadoop Yet-Another-Resource-Negotiator (YARN) Architecture

\subsubsection{Apache Hadoop Yet-Another-Resource-Negotiator (YARN)}

YARN is based on idea of splitting JobTracker's responsibilities for resource management and job scheduling/monitoring between two daemons. YARN replaces JobTracker by the global ResourceManager, which communicates with per-application ApplicationManagers to allocate resources for the particular application. Moreover, ApplicationManager monitors the execution of the tasks via communication with individual NodeManagers, each of which is assigned to a particular node.

The allocation of the resources in YARN is based on the notion of container. The Container represents an allocated resource in the cluster. The Container incorporates resource elements such as memory, CPU, disk, and network. The ResourceManager is the sole authority to allocate any Container to applications. The allocated Container is always on a single node and has a unique Container ID. Each container has a globally unique Container ID, and stores the unique ID of the specific node it was allocated on, the NodeID. More over aContainer has Resource and Priority objects, which contains detailed information about the container's structure.

Containers are assigned to the application via a Resource Scheduler. YARN has a modular scheduler, which deals exclusively with the allocation on the resources in given priority to the applications. Different schedulers are provided by the Hadoop 
framework. The default YARN scheduler is the Capacity Scheduler, which is designed to run Hadoop applications as a shared, multi-tenant cluster in an operator-friendly manner while maximizing the throughput and the utilization of the cluster. Scheduler class can be changed via a yarn - site.xml configuration file. For example, the Fair Scheduler allows YARN applications to share resources in large clusters fairly.

A Resource calculator used by a scheduler could be chosen through the setting of Resource Calculator property in the scheduler configuration file. The default scheduler is based only on memory parameter, while the Dominant Resource Calculator uses chosen Dominant-resource to compare multi-dimensional resources such as memory and CPU. Note, that a scheduler in YARN does not perform monitoring or track the status for the application. Hence, a scheduler offers no guarantees on restarting of failed tasks. Moreover, a YARN system reuses an existent MapReduce network, which ensures compatibility.

\subsection{Hadoop on YARN}

The current YARN-based Hadoop is a complex multilayered ecosystem. It could be viewed in layers as shown in Figure 5.3. The first layer is the file system, responsible for storage detection of failures and quick automatic recovery. The file system is represented mostly by HDFS, which could be specialized with additional packages implemented on top of it, such as HBase. The next layer consists of YARN, which operates on top of the storage layer and is responsible for main logic and computations. YARN solely composes the cluster compute layer. YARN applications are built immediately on top of YARN and interact with it by mean of YARN API creating YARN applications layer. This architecture widen horizons of Hadoop computation models from MapReduce only to a virtually limitless number of Big Data programming concepts, which could be implemented within the Hadoop ecosystem. In addition to MapReduce, the most popular of the currently implemented YARN applications are Spark and Tez. On top of these applications, users can build their own applications, or go one level higher and use the second application layer, which consists of applications, which do not interact with YARN directly, such as Pig, Hive, Cascading, Crunch. 


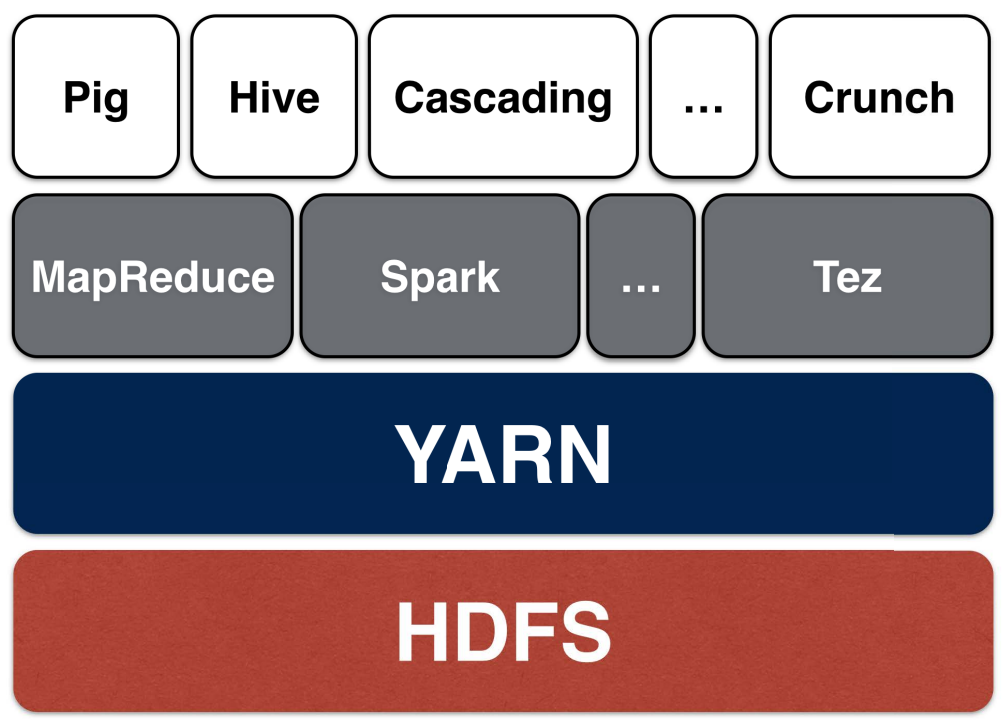

Figure 5.3: Apache Hadoop on YARN Structure

\subsubsection{YARN Application Execution Overview}

The application execution starts with a client contacting the Resource Manager, requesting an Application Master. An Application Master instance must run on one of the cluster nodes. In order to allocate resources for an Application Master, the Resource Manager contacts node managers and determines a node with available sufficient resources or, in other words, has a suitable container. Next, if an application is small and container resources are sufficient to complete the job, the application runs locally on the node. Otherwise, more resources are allocated. In order to acquire additional resources, an Application Master calculates the number and configuration of additional containers needed for the application, then requests them from the resource manager. These containers could be allocated on different nodes and an application will run on a distributed system. In the current paper, research concentrated on the 2.2 version Hadoop MapReduce. Therefore, let us consider a Hadoop MapReduce on YARN job in detail.

\subsubsection{MapReduce Job Execution Overview}

MapReduce job execution could be divided in four consecutive steps: job submission, job initialization, task assignment and task execution.

The first step is job submission; it starts from a call of a submit method of a 
Job instance. Within the submit method, a JobSubmitter instance is created and its submitJobInternal method is executed. After job submission, waitForCompletion method receives updates on job progress every second and outputs changes to the console. The JobSubmitter requests a new application ID (job ID) from the resource manager, checks the job's output specifications, computes the job's input splits, copies required for the job resources and, at last, submits the job by means of submitApplication method of resource manager.

The second step is job initialization, in which the resource manager delegates the request to a YARN scheduler, which allocated a container for the application master process, and the resource manager launches it under a node manager of the particular node. The application master (MRAppMaster class)retrieves previously computed input data splits and created map tasks, accordingly. Usually, a one map task per one split. Next, reduce tasks are created based on mapreduce.job.reduces property. All tasks are assigned a globally unique task ID.

The application master determines if this job uberized and can be completed within the same JVM, sequentially. If not, additional containers will be requested in the next step.

The third step is task assignment. First, requests for all map tasks are directed to the resource manager. Requests for reduce tasks issued after at least $5 \%$ of map tasks were completed. By default, each task is allocated 1024MB of memory and one virtual core. These parameters could be modified by means of configuration settings: mapreduce.map.memory.mb, mapreduce.map.cpu.vcores, mapreduce.reduce.memory.mb, and mapreduce.reduce.cpu.vcores. These settings could be configured for each individual job and limited by minimum and maximum settings set for the YARN cluster configuration. By means of a YARN scheduler, the resource manager assigns specified containers to the tasks and returns the list of these containers to the Application Master.

The forth step is task execution. The Application Master contacts the node managers of the nodes, which have the assigned containers and start the containers. Tasks are instances of the YarnChild class. The next task localizes all necessary resources and then has the map or reduce task executed. 


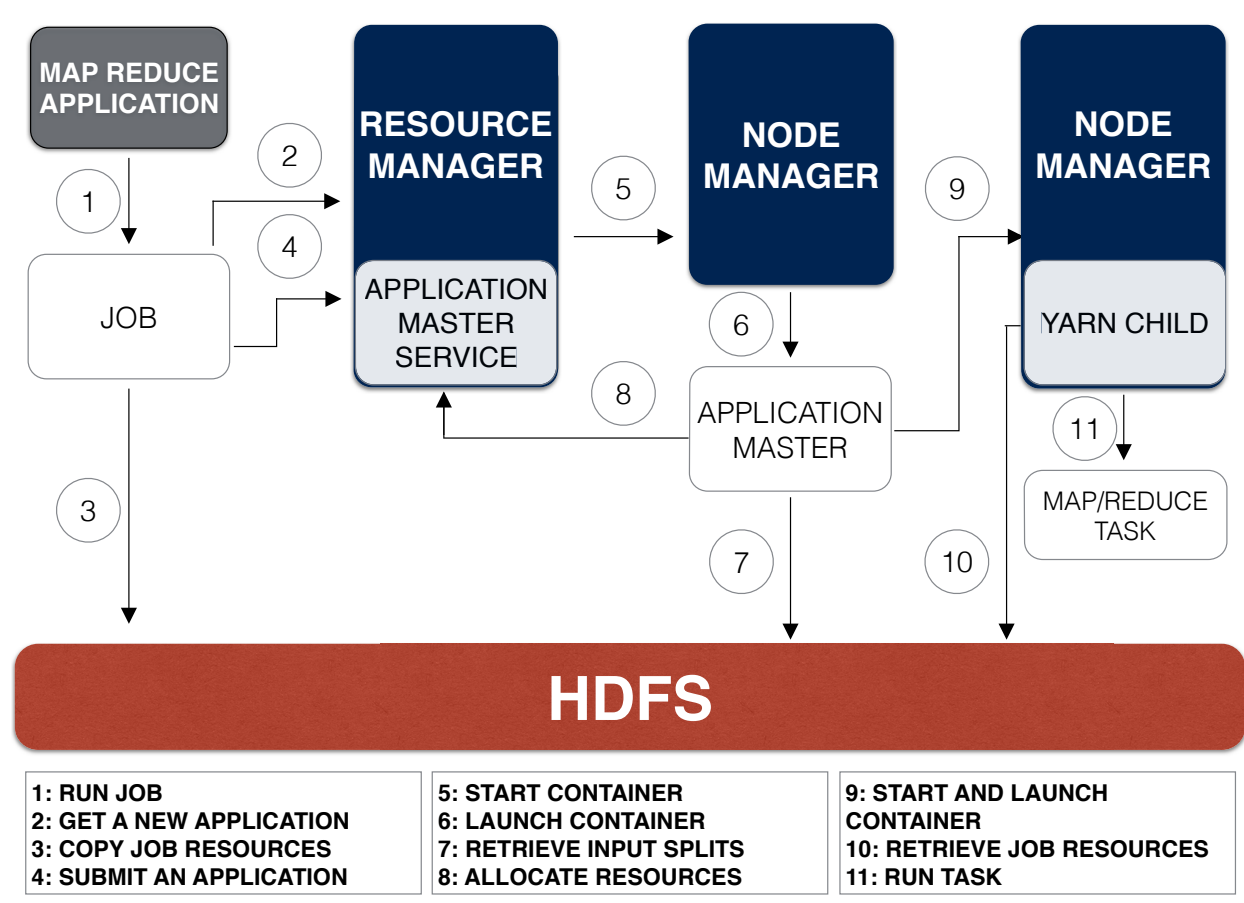

Figure 5.4: MapReduce Jod Run on Apache Hadoop on YARN

\subsection{Detailed Exploration of the Current Imple- mentation of the Most Relevant Packages}

\subsubsection{Resource Manager}

The Resource Manager is a key process, which controls all available cluster resources through collaboration with Application Masters and Node Managers. Application Masters negotiate resource allocation with the Resource Manager in order to obtain resources necessary for the completion of the particular application. a Node Manager manages resources available on the single node according to the instructions given by the Resource Manager.

The Resource Manager has an elaborated structure containing multiple components. Crucial processes could be divided in five main classes: components interfacing the Resource Manager to the clients, components connecting the Resource Manager to the nodes, components interacting with Application Managers, Token security related components and Scheduler related components. The last group could be considered the core of the Resource Manager. 


\subsubsection{Scheduler Related Components}

Scheduler-related components are the heart of the Resource Manager and carry out the main functionality which is supported by the rest on the modules. The most significant classes of this group are ApplicationsManager, ApplicationACLsManager, ApplicationMasterLauncher, YarnScheduler and Container AllocationExpirer.

ApplicationsManager orchestrates all the submitted applications, including completed applications, and responds to all users requests made via command line interface. ApplicationACLsManager responds to the restricted administrator and authorized client requests, and maintains and enforces the Access Control Lists (ACL) per each application. ApplicationMasterLauncher is responsible for maintaining a thread pool to launch new Application Masters, and is responsible for cleaning up on Application Master termination. The YarnScheduler is responsible for allocating resources for all current applications taking into account to cluster resource limitations. Container AllocationExpirer controls the appropriate launch of all allocated containers on the corresponding nodes in order to optimize cluster utilization.

\subsubsection{Client Interfacing Components}

This group composed of two services dedicated to two types of users: Client Service

and Admin Service. Client Service is a client interface available to all users, which handles all RPC requests, such as application submission, application termination, obtaining queue information, and cluster statistics. Admin Service is a special interface dedicated to give high priority to requests issued by the operator. Hence, important operations, such as the refreshing node list or the queues configuration, are not waiting in the same queue as routine users requests.

\subsubsection{Connecting to the Nodes Components}

The Resource Tracker Service is the component that responds to Remote Procedure Calls (RPC) from the nodes. It facilitates the registration of new nodes, rejection of the requests from any invalid or decommissioned nodes, collection of the node heartbeats and redirection of this information to the Scheduler. The Resource Tracer Service intensively interacts with other two components of the Resource Manager, which also belong to this category: Node Manager Liveliness Monitor and Nodes List Manager. 
A Node Manager Liveliness Monitor tracks live nodes and notifies the Resource Manager of the dead nodes. The Node Manager Liveliness Monitor achieves that by monitoring individual nodes heartbeat time. If the time that passes since the nodes last heartbeat is greater than the configured interval of time, the node is marked dead and is expired by the Resource Manager. Moreover, all the containers currently running on an expired node are marked as dead and no new containers are scheduled on it.

The Nodes List Manager is a collection of valid and excluded nodes. Based on the host configuration files specified via yarn.resourcemanager.nodes.include-path and yarn.resourcemanager.nodes.exclude-path, the initial list of nodes is created. During Hadoop run, the Nodes List Manager is also responsible to move decommissioned nodes from the included nodes list to the excluded nodes list.

\subsubsection{Application Masters' interacting components}

The Application Master Service is the key component that handles responses to RPCs from all the Application Masters. The Application Master Service is responsible for wide variety of tasks, such as the registration of a new Application Master, controlling container allocation and deallocation requests from all running Application Masters, forwarding them to the Scheduler and processing termination and unregister requests from a finishing Application Master. The application Master Service works closely with an Application Master Liveliness Monitor, another Application Master communicating component.

An Application Master Liveliness Monitor is a component that assists the management of the list of Application Masters and their statuses. An Application Master Liveliness Monitor monitors each Application Master and its last heartbeat time. Similarly to the node management scheme, if an Application Master does not produce a heartbeat within a configured interval of time it is marked as dead and is expired by the Resource Manager. Next, all the containers currently running or allocated to that particular Application Master are also marked as dead. After that, the Resource Manager schedules the same Application Master to run on a new container. The number of such restarts is limited by configurable value. 


\subsection{Application Master}

An Application Master is a component responsible for the orchestration of the single job. An Application Master is initiated when a job started. Hence, multiple instances of the Application Master class could coexist. A user is provided with ApplicationMaster and MRAppMaster classes. The second class is designed to serve Map Reduce type of jobs. Moreover, a user is given functionality to create custom Application Master extending Application Master interface. Nevertheless, the main Application Master functionality must be supported in all variations.

When an application initiated the Resource Manager invokes the Application Master Launcher method, which requests container for an Application Master process. When it obtains the container, it starts an Application Master process in it and records bookkeeping information about the newly created Application Master in the Resource Master. Next the Application Master registers itself with the Resource Manager. The registration process includes assigning host ports, job status and job history tracking URLs, etc.

During application execution, an Application Master produces regular heartbeats to inform the Resource Manager of its condition. The heartbeats are an important part of fault resistance and the recovery mechanism. Moreover, the heartbeat mechanism is facilitated by an allocation request protocol (ApplicationMasterProtocol.allocate in org.apache.hadoop.yarn.api.protocolrecords.AllocateRequest). An Application Master requests, from the Resource Master, a number of containers with specified memory capacity operating on a particular number of virtual cores. These values are consistent throughout the execution and are calculated by Hadoop based on configuration files. Moreover, the Application Master could specify a locality request and a level of priority should be given to the location request. The Resource Manager responds with a set of newly allocated containers, completed containers and a current state of available resources. Note that each request includes all the containers necessary for the completion of the job; hence, the returned set of values just partially fulfills the request, in most of the cases. A Resource Requests communicated through the Application Master Service class of the Resource Manager.

Allocated containers are processed by an Application Master by means of the Container Launch Context method, which specifies container ID, local resources required by the executable, the environment to be setup for the executable, commands to execute and other specifics for each container. Next, a launched container is submitted 
to a Start Container Request method in the Container Management Protocol, which executes previously set commands and starts the container.

The control of the containers is facilitated by querying of the Resource Manager via an Application Master Protocol allocate method or via Container Management Protocol by querying for the status of the allocated container's ContainerID. On the job completion, the Application Master sends a Finish Application Master Request to the Resource Manager, which unregisters the Application Master and initiates the application completion process.

MRAppMaster, in addition to the previously described functionality, has a history server and segregates requests for a map and a reduce container. The reduce containers request is delayed, and the size of the delay is set by configuration. On initialization, the MRAppMaster retrieves all the input splits from file system. Then, the Application Master computes all the map tasks for each input split, while the number of reduce tasks is determined by the configuration. Upon job completion, the job history server archives all job information.

\subsection{YARN Schedulers}

A YARN Scheduler allocates resources to applications according to a specific policy. Hadoop 2.2 is distributed with 3 built in YARN Schedulers: FIFO, Fair and Capacity Scheduler. Moreover, a user provided with an extendable API for the cases when the custom Scheduler is required. Specifics of each of the Hadoop YARN Schedulers are described below.

\subsubsection{The FIFO Scheduler}

FIFO (First In First Out) is the simplest of the schedulers; it follows a well-known scheme by placing applications in a queue, then running them in the order of submission. Therefore, first, all requests for the first application are allocated, and only after that are requests for the next one considered. The strength of the FIFO approach is its simplicity and absence of additional configuration settings. Nevertheless, its performance is not ideal and it is not recommended for a shared cluster. For example, if a small job is submitted to a FIFO scheduler after a large one, it will not be served until the large job is run to completion. 


\subsubsection{The Capacity Scheduler}

The Capacity Scheduler requires a configuration file, which determines a hierarchy among users or organizations. Each organization is allocated a certain part of the overall cluster capacity and assigned a designated queue. Users within organizations could be farther divided in groups with different priorities. Inside the single user group tasks follow the FIFO schedule. Overall, the Capacity Scheduler supports multiple requests, provides security, elasticity, resource-based scheduling, and configurable job priorities. Hence, it provides an administrator with a number of fine tuning mechanisms to optimize cluster deployment in a multi-tenant environment.

\subsubsection{The Fair Scheduler}

The Fair Scheduler is also recommended for resource sharing on a large multi-tenant Hadoop cluster. The Fair scheduler assures that all jobs get an equal share of the total cluster resources. In the event of the job number change, when a job is completed or a new job is initiated, the fair scheduler redistributes the resources according to the recalculated proportions. Hence, the cluster is used to full capacity at any given moment. The downside of the method is the delay in obtaining new resources, while resources are released and room made for the new job pool. The fair scheduler could be fine-tuned with job priorities, which are used as weights to determine the fraction of total resources that should be made available to a job. A number of parameters could be provided to a Fair Scheduler by means of the configuration file, including enabling preemption, pool name property, allocation file name, and per job load manager.

\subsection{Tunable Hadoop Parameters}

Hadoop exposes hundreds of parameters and a small number of them have an extremely high impact on the performance and could not be derived from a static formula. Currently, the parameters must be obtained through manual iterative tuning. Therefore, only one set of parameters could be tested simultaneously. Information about tunable parameters is scattered among multiple sources such as white papers, Hadoop, Hortonworks and Cloudera web sites, and blog posts of credible professionals. Parameters that have a great impact on the cluster resource utilization and total execution time could be divided in four main categories: MapReduce related, YARN 
specific, HDFS related and core settings, which influence general configuration.

The first category includes mapreduce.job.reduces, mapred.child.java.opts, mapreduce.map.output.compress, mapreduce.map.cpu.vcores, mapreduce.reduce.cpu.vcores, mapreduce.reduce.memory.mb, mapreduce.map.memory.mb. The second category includes yarn.nodemanager.resource.memorymb, yarn.nodemanager.resource.cpu-vcores, yarn.nodemanager.resource. npercentage-physical-cpu-limit, $\quad$ yarn.scheduler.maximum-allocation-mb, yarn.scheduler.minimum-allocation-mb, yarn.scheduler.maximum-allocationvcores, yarn.scheduler.minimum-allocation-vcores. The third category includes dfs.datanode.handler.count, dfs.replication, dfs.blocksize. Finally, the fourth category includes io.file.buffer.size and file.blocksize.

The parameters that are responsible for the number and size of YARN containers for map tasks are mapreduce.map.memory.mb (mapred-site.xml) and mapreduce.map.cpu.vcores (mapred-site.xml), mapreduce.reduce.memory.mb (mapredsite.xml), mapreduce.reduce.cpu.vcores (mapred-site.xml). This list of parameters was selected based on previous Hadoop automatic tuning efforts and currently recommended for tuning parameters on the Apache Hadoop official web page [38] and the Hortonworks web page [39]. Previous work on Hadoop and MapReduce automatic tuning supports the given subset of the parameters as well as displays the necessity of autotuning these particular parameters and their combinations [8, 9, 20,21, 26-29]. The description of the parameters, the configuration file they are set in and the default values are presented in detail in Table 5.1. 
Table 5.1: The Default and Rules of Thumb (RoT) settings for Hadoop Parameters

\begin{tabular}{|c|c|c|c|}
\hline Name & Default & RoT & Description \\
\hline mapreduce.job. & 1 & 18 & The default number of \\
\hline reduces & & & reduce tasks per job. \\
\hline mapred.child. & $-\mathrm{Xmx} 200 \mathrm{~m}$ & $-\mathrm{Xmx} 860 \mathrm{~m}$ & Java opts for the \\
\hline java.opts & & & task processes. \\
\hline mapreduce.map. & FALSE & FALSE & Turns on or off compression \\
\hline output.compress & & & of the outputs of the maps. \\
\hline mapreduce.map. & 1 & 1 & The number of virtual \\
\hline cpu.vcores & & & cores required for each map task. \\
\hline mapreduce.reduce. & 1 & 1 & The number of virtual \\
\hline cpu.vcores & & & cores required for each reduce task. \\
\hline mapreduce.reduce. & 1024 & 1024 & Larger resource limit \\
\hline memory.mb & & & for reduces. \\
\hline mapreduce.map. & 1024 & 1024 & Larger resource limit \\
\hline memory.mb & & & for maps. \\
\hline yarn.nodemanager. & 8192 & 26000 & Amount of physical memory, \\
\hline \multirow[t]{2}{*}{ resource.memory-mb } & & & in $\mathrm{MB}$, that can be allocated \\
\hline & & & for containers. \\
\hline yarn.nodemanager. & 8 & 4 & Number of vcores that \\
\hline resource.cpu-vcores & & & can be allocated for containers. \\
\hline yarn.scheduler. & 8192 & 4000 & The maximum allocation for \\
\hline
\end{tabular}


Table 5.1 - Continued

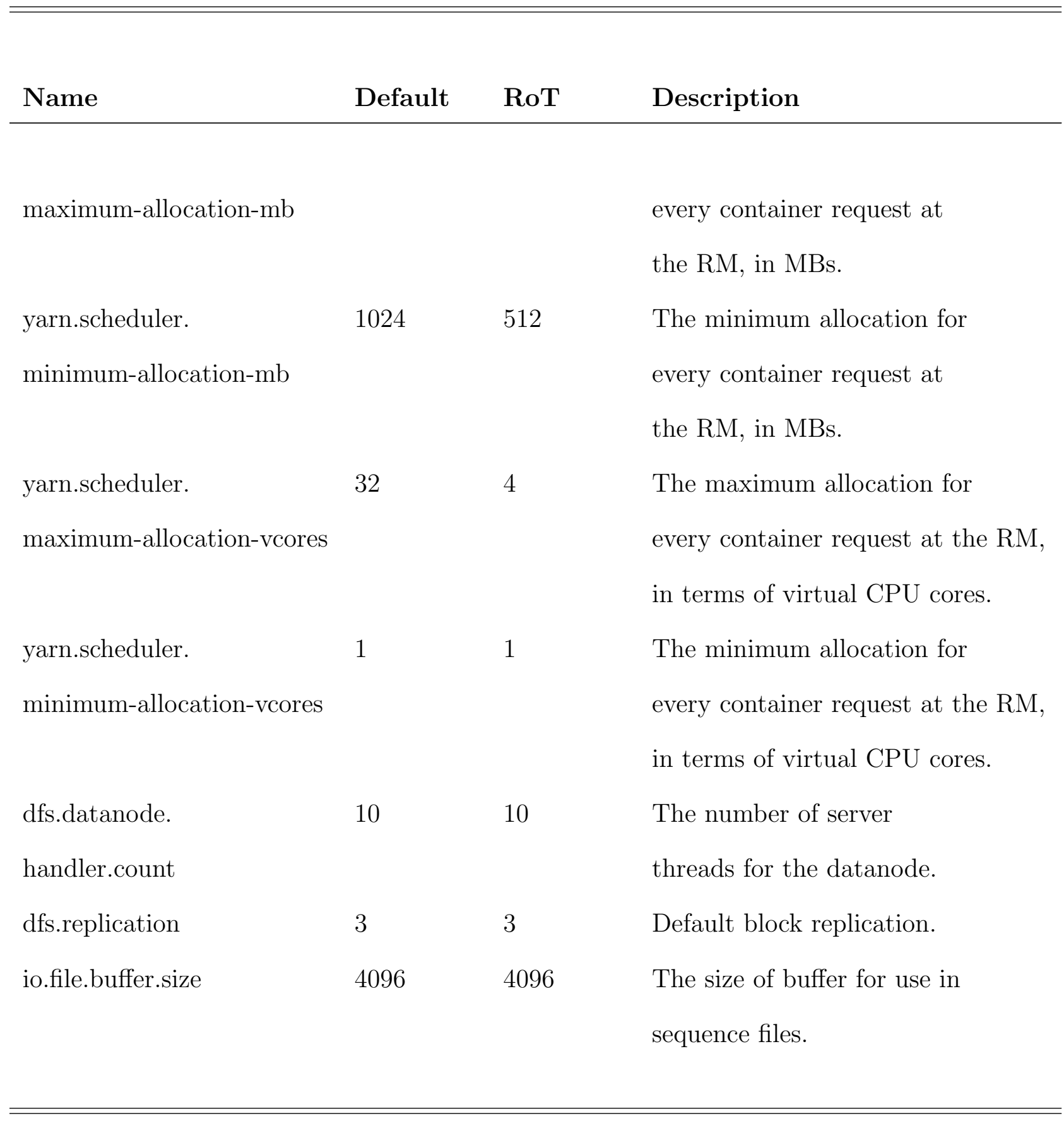




\section{Chapter 6}

\section{Autotuner Implementation}

\subsection{Overview of the Autotuner Placement Within the Hadoop}

The parameters selected for automatic tuning directly influence the structure of an individual container, in particular, the size of the container's memory and the number of virtual cores assigned per container. These parameters are read into the job configuration object by means of a proto mechanism. Next, when an application is initiated, a container request formed by the Resource Manager Application Launch mechanism for the first container, and in Application Manager for the remainder of the containers. All resource requests are passed asynchronously to the Application Master Service as shown in Figure 6.1. The Application Master Service directs the requests to the YARN Scheduler. A YARN Scheduler partially fulfills the resource requests by providing a number of the containers with memory size and the number of virtual cores specified in the Resource Request. Note that most of the requests receive a smaller number of containers than was requested. IDs of the allocated containers are returned to the Application Master Service, stored for bookkeeping purposes, and passed to the Application Master within the container allocated event. Moreover, information about completed containers is also passed via response to a Resource Request. Later, an Application Master starts a Java virtual machine on each of the allocated containers with a specified task. Allocated containers are taken into account

for the next resource request. Resource requests are sent out on a heartbeat, even if no more containers are required. In that case, the Resource Request with the number of required containers equaling zero is issued. 


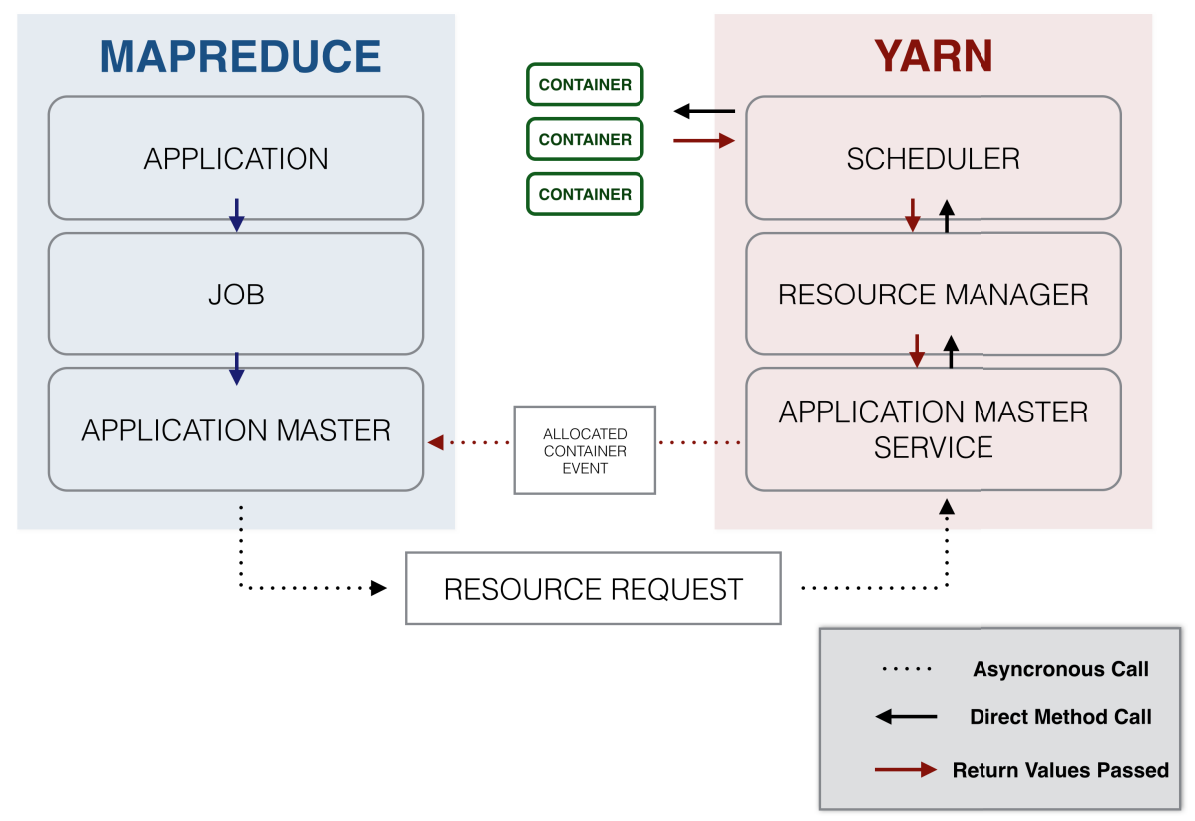

Figure 6.1: Hadoop YARN Resource Requesting Scheme

The autotuner connects to YARN machinery by intercepting the resource requests at the Application Master Service (Figure 6.2). The autotuner reads in a request and passes it to the tuning algorithm. Moreover, the autotuner provides the statistics for the algorithm, which associate previously used parameters with the cost. In the current implementation, cost is the average container completion time for all completed containers allocated with the given parameter set. The developed autotuner design is modular and could be easily extended to implement a variety of algorithms. At the moment, the autotuner includes the Hill Climbing and Explorer algorithm implementations. After receiving the statistics, an algorithm provides a new parameter set and passes it to the autotuner. The autotuner calculates number of containers for the parameter set, composes a new resource request and passes it back to the Application Master Service, which proceeds with the allocation method. Newly allocated and completed containers are added to the autotuner statistics. When all job tasks are completed, the Application Master Service unregisters the Application Master and an autotuner outputs complete wave statistics into a log file. 


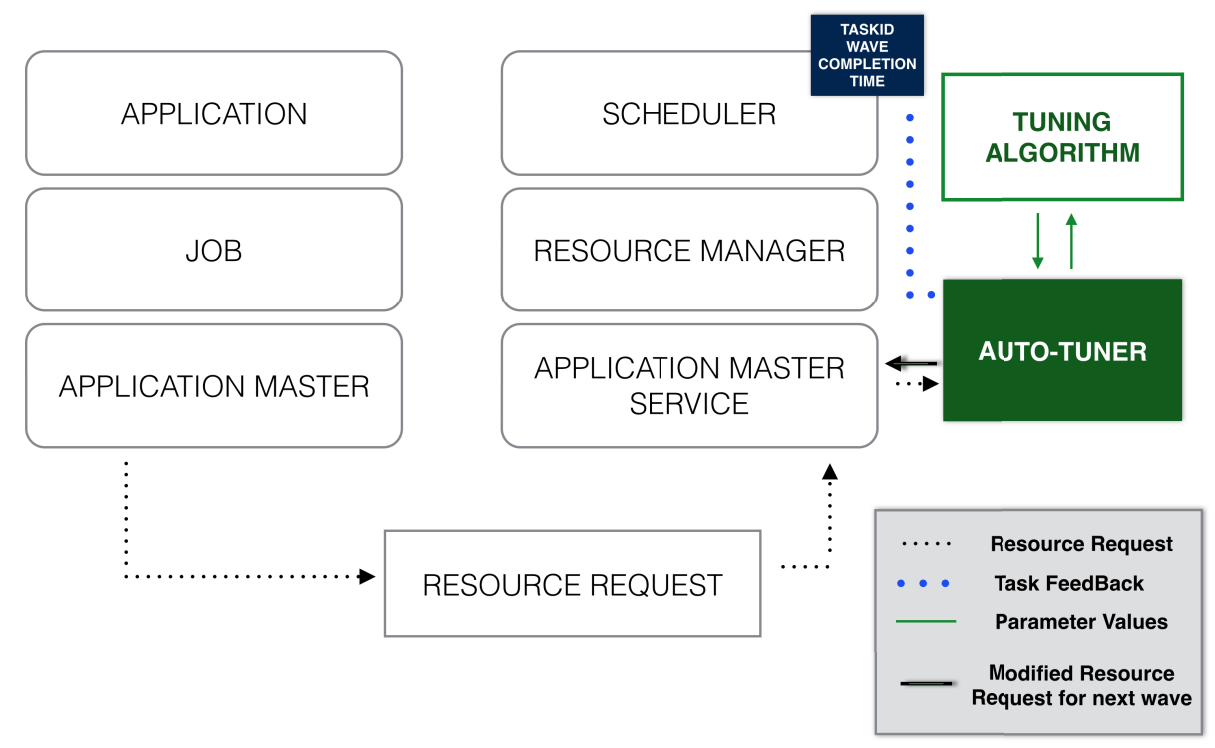

Figure 6.2: Autotuner Placement in Hadoop YARN

\subsection{Autotuner Implementation Details}

\subsubsection{Autotuner Classes}

During the initiation of the Application Master Service, an Autotuner instance is also created. An Autotuner object includes a number of attributes, including an instance of the selected type algorithm (Algo algo) and an autotuner wave record-keeping object (WaveMetrics waveMetrics).

All algorithms must extend a general tuning algorithm class Algo and implement toTune function, which takes in a Parameters object and returns a Parameters object. A Parameters object currently has two attributes: vCores and memory. The Parameters class could be easily extended to include more parameters. In addition, the number of containers in the original Resource Request is included in a form of parameter(numCont). Moreover, the class provides all necessary public get and set methods for the attributes.

Three algorithm classes (Algo extensions) were implemented: HCAlgo, the classic hill climbing algorithm implementation, and two variations of Explorer, the testing algorithm used for the exploration of a parameter space (ExpAlgo and ExpAlgo2).

HCAlgo includes a boolean fixed which indicates if the stopping condition was 
met. The stopping condition is the absence of a neighbour with a lower cost. Once the stopping condition is met fixed is set to true and the Parameters object stored in bestPar and, from this moment to the job completion, bestPar is always returned on toTune() calls. If the fixed variable is still false, than the remaining neighbours of the current parameters (currentPar) are explored. A new Parameter set is created by an increase or decrease of memory or vCores and the results are stored in candidatePar. A selection between which of the parameters will be altered, and in what direction, is based on a random integer. Note that the parameter space has different length dimensions, as the number of the valid virtual cores per container is much smaller than the number of possible values for a container memory size. The Explorer algorithm loops until all parameters within the set range are explored; it does not have a stopping condition. An Explorer collects statistical information for each wave and outputs it to the log file on completion of the job in the finishApplicationMaster method of the Application Master Service.

Statistics on autotuner waves are stored in a waveMetrics object, which includes a map, contWave of all allocated containers, ContainerIDs, a wave ID and a list of the all created Wave objects. Each Wave object includes an integer wave ID (waveId), and boolean newTimeAdded, which indicates if new time was added. Moreover, it keeps track of all completed containers of the wave in a contIDs array list. The average time of the wave (averTime) is calculated only if it was requested and if a new container completion time was added since the last average time calculation. Such a "lazy evaluation" scheme saves from unnecessary average time calculations. Relations between autotuner waves and Hadoop waves are discussed in next section.

\subsubsection{Autotuner Waves}

A Hadoop wave is a collection of all containers returned as a result of a single request. Therefore, all containers of a Hadoop wave started at the same time and have the same memory size and number of virtual cores. A Hadoop wave is created on every Hadoop heartbeat. Moreover, an autotuner wave collection of all containers is returned as a result of all resource requests emitted within the same autotuner wave. An autotuner wave is created after a set amount of time is passed, which typically includes multiple heartbeats. An Autotuner Wave includes one or more waves initiated within a set time period. Only full waves are included. Hence, no wave could have containers in two separate Autotuner Waves. To illustrate the relations between the wave types 


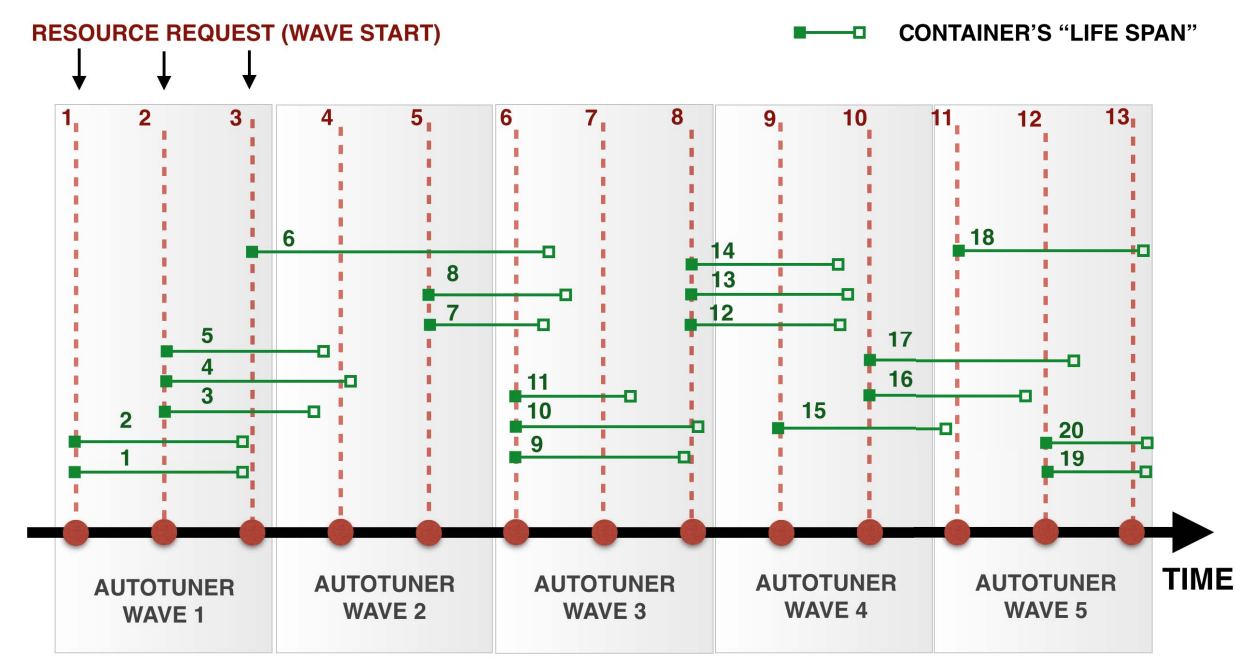

Figure 6.3: Autotuner Waves

Figure 6.3 shows a schematic example of a single job execution with 20 containers created within 13 Hadoop waves. These waves are dispersed among five autotuner waves.

\subsubsection{Execution order of the Autotuner}

On each heartbeat, a resource request is sent from the Application Master to the Resource Manager. The Application Master Service within the Resource Manager receives resource request in its allocate method and passed it to the autotuner. If conditions for a creation of a new wave are met, parameters of the resource request are passed to the autotuner's toTune method. The method validates the request and sends it to an algorithm's (Algo) toTune method. This method returns a Parameter object containing new parameters for the resource request. Autotuner validates parameters, creates a new wave, adds it to statistic records, and sends out a modified request to the scheduler. If no new wave is created, then a resource request is composed according to current wave specs and is passed to a scheduler as part of the previously created wave. The response to a request returned from the scheduler contains containers IDs of allocated containers for a wave and information on completed containers. This information is added to the wave records. 


\subsubsection{Explorer}

An Explorer was created to evaluate the variation between completion times of the autotuner waves with different parameter settings. Two versions of this algorithm were used in experiments: ExpAlgo and ExpAlgo2. Both of the versions extend the Algo class and implement a toTune function.

ExpAlgo has an autotuner wave length of 30 seconds and considers a two dimensional subspace of virtual cores (vCores) and memory. The number of virtual cores varies between 1 and 4. Container memory ranges from $640 \mathrm{MB}$ to $3200 \mathrm{MB}$ and includes 6 preset values, one of which is the default.

ExpAlgo2 has an autotuner wave length of 20 minutes and considers a one dimensional parameter subspace of memory. The number of virtual cores is fixed to 1 . Container memory ranges from $640 \mathrm{MB}$ to $3200 \mathrm{MB}$ and includes the same 6 values as the ExpAlgo. 


\section{Chapter 7}

\section{Results}

\subsection{Experimental Design}

Testing was completed in three different settings: default baseline, rules of thumb baseline and auto-tuned. Testing included two baselines. The first was the execution of TeraSort, included in the standard Hadoop distribution for 100GB and 1TB of data. The second was the completion of TPCxP Benchmark for 1TB of data.

For the default baseline, tests were executed on "out of the box" with default settings for all Hadoop 2.2 parameters. For values of the important subset of parameters see Table 7.1. Rules of thumb (ROT) were collected from multiple sources. The subset of the most important parameters, which are suitable for Hadoop 2.2 were selected and displayed in the Table 7.1. These settings were used for the rules of thumb baseline. Finally, the third set of tests was performed on the system with ROT

values for the listed parameters changing tunables (mapreduce.map.memory.mb and marpeduce.map.cpu.vcores from mapred - site.xml) via the autotuning mechanism.

\subsubsection{Testing Cluster Configuration and Hardware}

The cluster is composed of four identical machines, three of which are data nodes (slaves) and the remaining one is a master node.

Each of the computers is empowered by Intel(R) Core(TM) i7-3770 CPU @ $3.40 \mathrm{GHz}$ with $31 \mathrm{~GB}$ of memory core. In addition to the 120GB INTEL solid-state drive, which contains the operating system, it has four additional 1863GB drives, which, all together results in a 5.37 TB storage capacity per each machine (node). 


\subsubsection{Hadoop Cluster Settings}

Configuration files were altered in a following way. First, hdfs-site.xml composed of four properties: fs.defaultFS, dfs.datanode.data.dir, dfs.replication and dfs.namenode.name.dir with hdfs://ivy23/, /hadoop2,/hadoop3,/hadoop4, 3 and /hadoop1 values, respectively. Second, core-site.xml composed of two properties: fs.defaultFS and hadoop.tmp.dir set to hdfs : //ivy23/ and /hadoop $1 /$ tmp values, respectively. Third, mapreduce-site.xml has a single parameter mapreduce.framework.name with value yarn. Fourth, yarn-site.xml has three original parameters: yarn.resourcemanager.hostname is set to the master node (ivy23 in the particular test cluster), then yarn.nodemanager.aux services and yarn.nodemanager.aux - services.mapreduce.shuffle.class are set to mapreduceshuffle and org.apache.hadoop.mapred.ShuffleHandler values, respectively.

Additional configuration files (hadoop - policy.xml, httpfs - site.xml, kms site. $x m l)$ remained unaltered.

\subsubsection{Development environment set up with MiniYARNclus- ter on Eclipse development environment}

The configuration of the development environment itself was a nontrivial task; therefore, the setting up procedure presented in detail. The Hadoop 2.6 source code was downloaded from the Hadoop official web page [12]. Next, Eclipse Platform Version: 3.8.1 for Java Developers was installed on the Ubuntu 14.04 operating system. Moreover, the Apache Maven 3.0.5 plugin was installed for a building process.

In order to trace the YARN Resource Manager actions, a MiniYARNCluster type of execution must be configured. MiniYARNCluster simulates real Hadoop/YARN cluster communication among the nodes and architectural modules by initiating multiple Java virtual machines (JVMs).

\section{Configuration}

Configuration files are found in /hadoop-2.6.0-src/hadoop-yarn-project/hadoopyarn/hadoop-yarn-server/hadoop-yarn-server-resourcemanager/src/test/resources.

A YARN configuration file yarn-site.xml must be modified. In particular, by default, the configuration file does not contain any properties. A YARN configuration 
file yarn-site must be modified. Between configuration, tags must be added two properties: yarn.nodemanager.aux - services with MapReduce shuffle value, and yarn.nodemanager.aux - services.mapreduceshuffle.class property with org.apache.hadoop.mapred.ShuffleHandler value.

\section{Example file}

In the example Java file before the actualMapReduce job is run, a cluster must be set up and started and, after a MapReduce job is complete, it must be stopped. The code must be added to the main class, before the example code. The next five steps complete the configuration.

1. First logging should be set

Log LOG = LogFactory.getLog(WordCount.class);

LOG.info("Starting up Mini YARN cluster);

2. Next, the new configuration is created and the MapReduce framework parameter is set to YARN. Moreover, additional parameters, such as minimum allocation for a scheduler could be set if default values do not suffice.

Configuration conf $=$ new Configuration () ;

conf.set("mapreduce.framework.name", "yarn");

conf.setInt(YarnConfiguration.RM SCHEDULER MINIMUM ALLOCATION $\mathrm{MB}, 64)$;

3. After the mini cluster is initiated and started.

MiniYARNCluster miniCluster = new MiniYARNCluster("testYarnCluster",1,1,1);

miniCluster.init(conf);

miniCluster.start();

4. It is important to create and pass to the mini cluster URL for yarn-site.xml YARN configuration file and process the parameters that it contains.

URL url = Thread.currentThread().getContextClassLoader().getResource ("yarn-site.xml"); 
if (url $==$ null) \{

throw new RuntimeException("Could not find 'yarn-site.xml' dummy file in classpath");

$$
\}
$$

miniCluster.getConfig().set("yarn.application.classpath",

new

File(url.getPath()).getParent());

OutputStream os $=$ new FileOutputStream(new File(url.getPath()));

miniCluster.getConfig().writeXml(os);

os.close ();

5. Finally, the configuration with the binding details of the created mini YARN cluster could be obtained.

YarnConfiguration appConf $\quad=\quad$ new
tion(miniCluster.getConfig ()$) ;$

This configuration should be passed to the app on job instantiation step. After job completion, the mini cluster should be stopped with miniCluster.stop() method.

The example constructed following the example given above could be executed in the development environment, such as Eclipse, by running in normal or debugger mode. In debugger mode, breakpoints could be used in order to trace the anatomy ofMapReduce job processing on a YARN cluster.

\subsubsection{Benchmarks}

Quantitative evaluation and characterization of an academic or commercial software product through extensive benchmarking is crucial. A benchmark not only places a compatible value on its performance but also directs development toward the most valuable improvements. A number of benchmarks different complexity levels were considered.

\subsubsection{TeraSort Benchmark}

\section{TeraGen}

TeraGen is a MapReduce program that produces a requested size file, which can be used as an input for the TeraSort benchmark. In particular, a user specifies a number 
of rows as a command line argument and a directory for the output. Each row consists of 100 bytes. The first 10 bytes are the key and composed of random characters from the set ' '.. '' '. The second 10 bytes are an integer row ID. Next 78 bytes are the filler, which consists of seven runs of 10 characters from 'A' to 'Z'.

TeraGen was executed from Hadoop home directory on the master node and test data was generated.

$$
\text { bin/hadoopjarhadoop - examples - *.jarteragen } 10000000000 / \text { terasort - input }
$$

\section{TeraSort}

TeraSort is a traditional MapReduce benchmark, taking its roots from the tests proposed in the original Google paper [5]. TeraSort is a MapReduce program, which samples input, and produces globally sorted output of the data. TeraSort differs from other MapReduce sorts by the custom partitioner. The partitioner utilizes the list of N-1 sampled keys. Therefore, reducer $i$ receives only keys in interval $[$ sample $[i-1]$; sample $[i])$. Hence, all keys separated in ordered "buckets", and output of $i$-th reducer is always greater than output of $(i-1)$-th. Indexing of the sample keys is based on the first two bytes of the key and implemented as a two level trie. Such structure speeds up the partitioning process.

Before a job is submitted, TeraSort generates the sample keys by sampling the input. The list of sample keys is written into HDFS. Note that, the output of the reduce has replication set to 1 , instead of the default 3 .

TeraGen was also executed on the data previously produced by TeraGen.

bin/hadoopjarhadoop - examples - *.jarterasort/terasort - input/terasort - out

\section{TeraValidate}

TeraValidate is a MapReduce program, which was developed for validation of TeraSort output. It confirms that produced rows are in globally sorted order. Each output file assigned to designated map task, which ensures that each key is less than or equal to the previous one and generates records with the first and last keys of the file. Reducer confirms that the first key of file $i$ is greater that the last key of file $(i-1)$. If this is not the case, a record is produced to the output. Therefore, an empty output signifies that data was sorted correctly. 


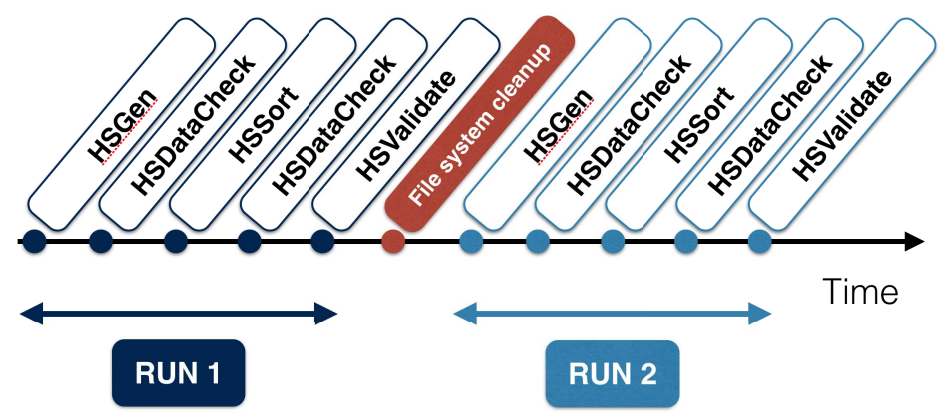

Figure 7.1: TPCx-HS Execution Phases

TeraGen was executed from the Hadoop home directory on the master node and $1 \mathrm{~TB}$ of previously sorted data was checked. bin/hadoop jar hadoop-examples-*.jar teravalidate /terasort-out / terasort-val

\subsubsection{TPCx-HS Benchmark}

In addition to the commonly used TeraSort a new yet highly reputable, industry grade benchmark, TPCx-HS was used. Transaction Processing Performance Council (TPC) is a non-profit organization, which defines collection of benchmarks for transaction processing and databases, which are widely accepted in academics and industry. All benchmarks separated in four groups: enterprise benchmarks, obsolete benchmarks, express benchmarks and other benchmarks. The benchmark chosen for the evaluation of the project is in the express category under the TPCx-HS benchmark tool set. TPCx-HS was released in August 2014 as the first vendor independent industry standard Big Data benchmark. The TPC big data committee membership includes major systems and software vendors such as IBM, Intel, HP, Microsoft, Oracle, Red Hat, and others. The TPCx-HS stresses both hardware and software including Hadoop run time, Hadoop File system API compatible systems and MapReduce layers.

The TPCx-HS benchmark workload consists of the HSGen, HSDataCheck, HSSort and HSValidate. A valid run will consist of five separate phases run sequentially. The elapsed time for each phase was recorded. The benchmark test consists of two runs. Note that no activities, except file system cleanup, are allowed between Run 1 and Run 2.

No manual tuning, reconfiguration or rebooting is permissible during the runs or between the runs. Automated changes and tuning performed between any of the 
phases are allowed, but must be disclosed. The TPCx-HS follows a stepped size model. The Scale factor (SF) used for the test dataset must be chosen from the set of fixed Scale Factors defined as follows: 1 TB, 3 TB, 10 TB, 30 TB, 100 TB, 300 $\mathrm{TB}, 1000 \mathrm{~TB}, 3000 \mathrm{~TB}$, and $10000 \mathrm{~TB}$. The corresponding number of records is as follows: $10 \mathrm{~B}, 30 \mathrm{~B}, 100 \mathrm{~B}, 300 \mathrm{~B}, 1000 \mathrm{~B}, 3000 \mathrm{~B}, 10000 \mathrm{~B}, 30000 \mathrm{~B}$, and 100000 $\mathrm{B}$, where each record is 100 bytes generated by HSGen. TPCx-HS produces four metrics: HSph@SF , the Performance Metric, \$/HSph@SF , the Price-Performance

metric, the System availability date and the TPC-Energy metric. The performance metric is calculated as:

$$
H S p h @ S F=\frac{S F}{(T / 3600)}
$$

, where $\mathrm{SF}$ is the Scale Factor and $\mathrm{T}$ is the total elapsed time in seconds.

Note that results could be compared only if the scale factor is the same.

\subsubsection{NMON}

Nigel's performance Monitor (NMON) in a popular system-monitoring tool for the Linux operating system [40]. NMON provides information on the CPU, memory, network, disks (mini graphs or numbers), file systems, NFS, top processes, resources (Linux version and processors) and on Power micro-partition in real time. It could be used in two modes. First, real time monitoring with graphical interactive display. Second mode is called spreadsheet output format and records all information in a table in the form of snapshots of the system state. The intervals for the snapshots and the total number of them are set by the user. For the current project, the second mode of the NMON was used with information recorded every minute for 60 hours. The command was executed on all nodes and composed files were processed with nmon analyzer tool, distributed as an open source project by Intel [40].

\subsection{Parameter Space Exploration}

In addition to the TeraSort and TPCx-HS benchmarks for baseline testing, the NMON monitoring system was used in order to indicate levels of the cluster resource utilization.

A group of tests with static values for the number of virtual cores and memory 


\begin{tabular}{ccccccc}
\hline Memory & Average & Test 1 & Test 2 & Test 3 & Test 4 & Std. Deviation \\
\hline 640 & 29 & 29 & 20 & 34 & 33 & 6.4 \\
1024 & 35 & 21 & 25 & 44 & 51 & 14.5 \\
1280 & 22 & 19 & 25 & 21 & 2 & 2.5 \\
1920 & 20 & 20 & 18 & 24 & 17 & 3.1 \\
2650 & 23 & 25 & 20 & 22 & 23 & 2.1 \\
3200 & 30 & 30 & 30 & 29 & 30 & 0.5
\end{tabular}

Table 7.1: Completion Times for TeraSort on 100 GB Data with Different Values of mapreduce.*.memory.mb Parameters with Calculated Standard Deviation

per container parameters was performed in order to confirm the impact of the parameters selected for tuning on the overall job execution time. Settings for this test were adjusted according to the Rules of Thumb (RoT) (Table 5.1). The MapReduce job chosen for the tests is TeraSort, which is included in Hadoop 2.6 examples folder, for 100 GB data generated by TeraGen, included in the same package. A subset of the parameter space between $640 \mathrm{MB}$ and $3200 \mathrm{MB}$ with three, equally spaced intermediate values, and the default value, were chosen for the tests. Table 7.1 displays obtained results and the standard deviation of the values for each memory setting. Obtained values are also visualized in the Figure 7.2 with the corresponding polynomial trend line. The trend line exposes non-unimodal behaviour with a local minimum at 640 $\mathrm{MB}$, the global minimum at $1920 \mathrm{MB}$ and the global maximum at $1024 \mathrm{MB}$, which is the default value (see Figure 7.2).

\subsection{Default Baseline}

\subsubsection{TeraSort}

The test establishing the default baseline was performed with "out of the box" settings, executing a TeraSortMapReduce job included in the Hadoop 2.6 examples package. 1TB Terasort execution with default settings was completed in 1 day 6 


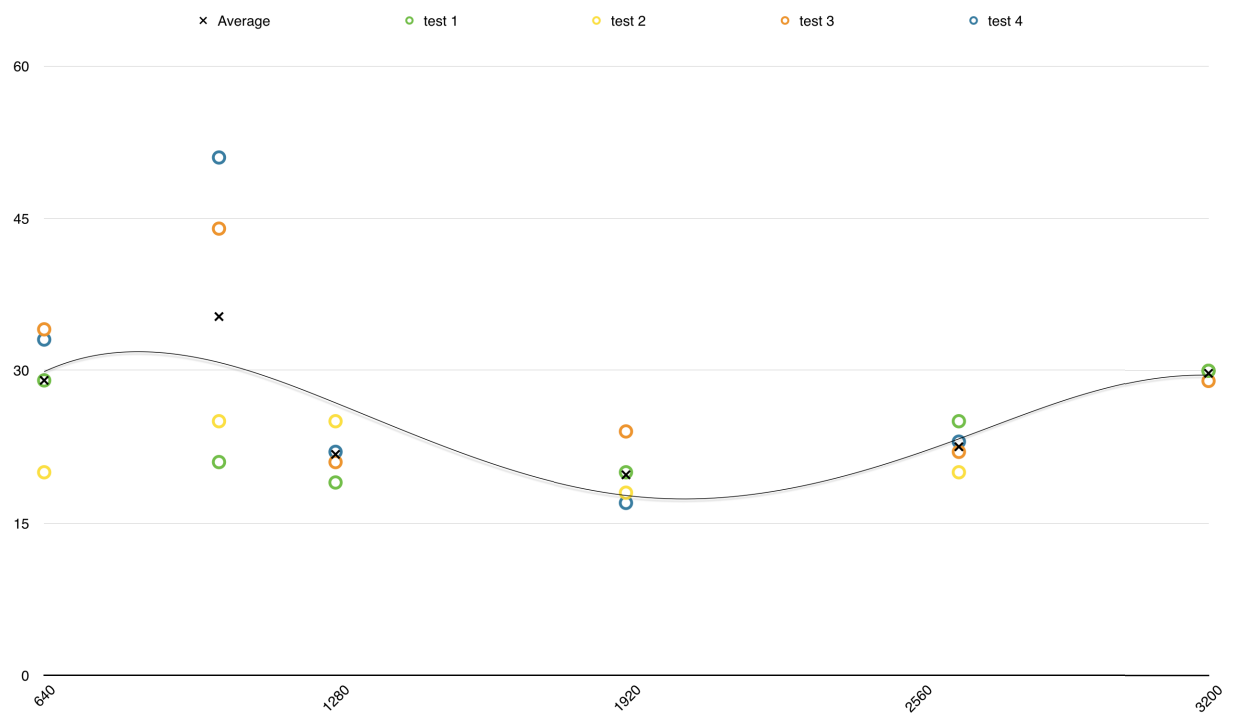

Figure 7.2: Graph of Completion Times for TeraSort on 100GB Data with Different Values of mapreduce.*.memory.mb Parameters

hours 55 minutes and 1 second. Moreover, the reduce stage execution took 1 day 6 hours 50 minutes and 52 seconds. Cluster resource usage was collected with nmon and processed with the nmon analyzer tool (Figure 7.3).In particular, the figure shows examples of Total CPU Utilization, CPU Utilization per CPU, and Disk I/O on a data node $(\mathrm{A}, \mathrm{B}, \mathrm{C})$, while Network I/O, and Network Packets activity are recorded on the master node $(\mathrm{D}, \mathrm{E})$. Note that all three data nodes exhibit practically identical resource utilization and activity; hence, the graphs constructed for one of the data nodes are sufficient to represent all data nodes in general.

\subsubsection{TCPx-HS}

Two tests establishing the TPCx-HS default baseline were performed with "out of the box" settings. Each of the tests composed of the two consecutive jobs, total time and HSph@SF measurement. Resulting values are shown in Table 7.2. Cluster resource usage was observed with nmon and processed with the nmon analyzer tool (Figure 7.4). In particular, the figure shows examples of Total CPU Utilization, CPU Utilization per CPU, and Disk I/O on a data node (A,B,C), while Network I/O, and 
A

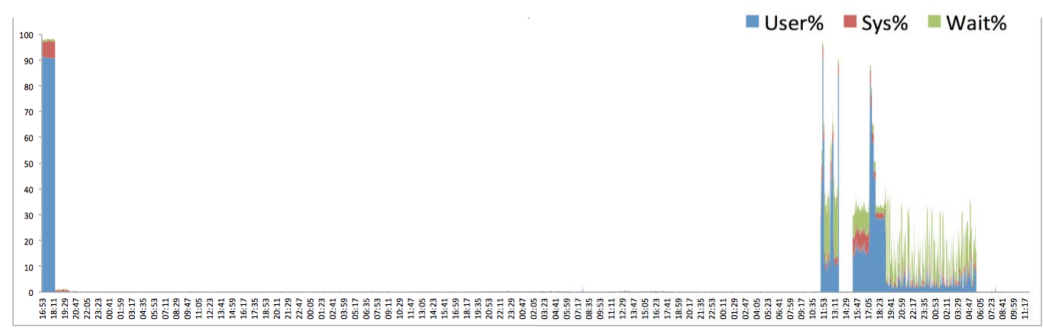

B

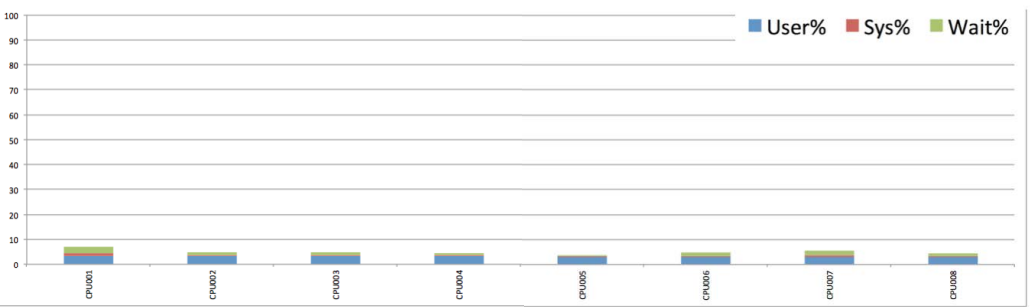

C

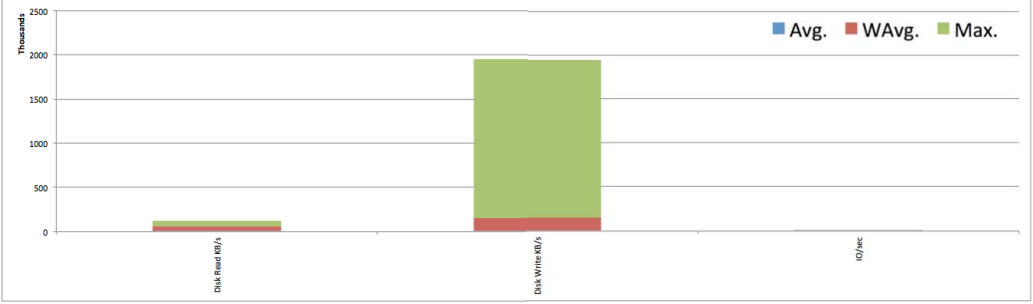

D

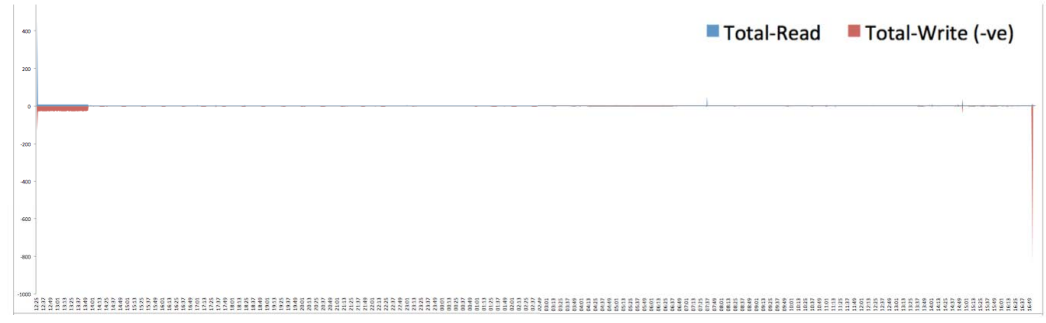

E

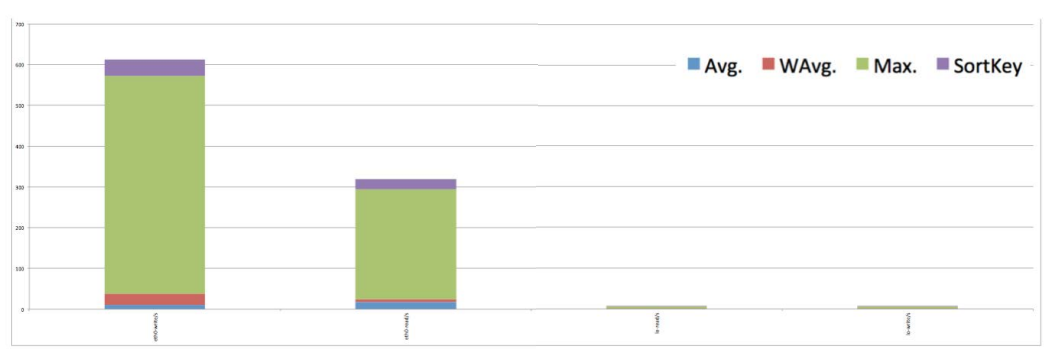

Figure 7.3: NMON Analyzer results for $1 \mathrm{~TB}$ Terasort test with default parameters. (A) Total CPU Utilization, (B) CPU Utilization per CPU, (C) Disk I/O, (D) Network I/O, (E) Network Packets. 
A

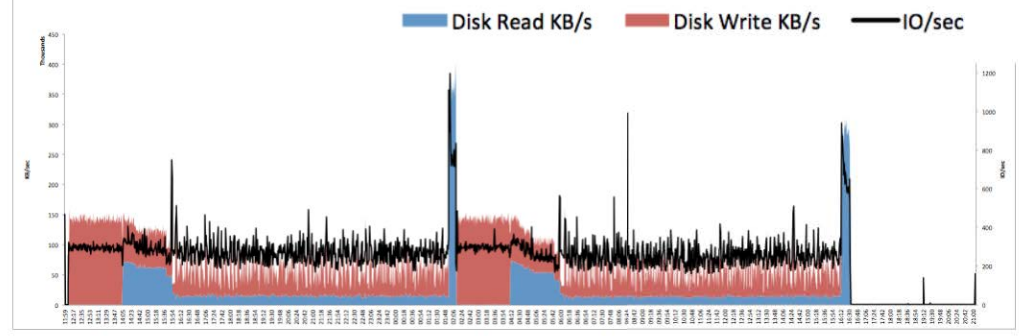

B

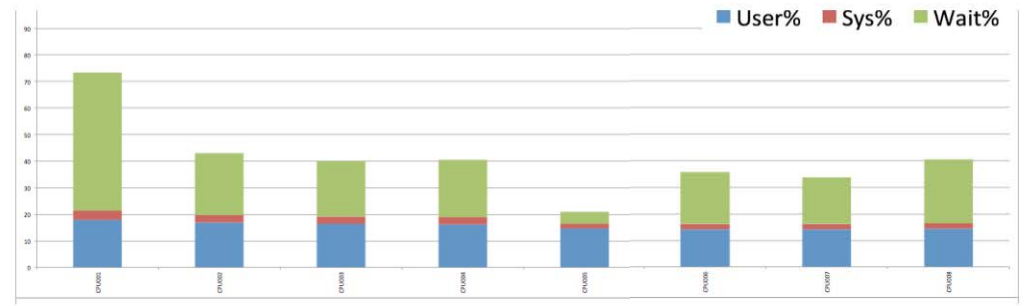

C

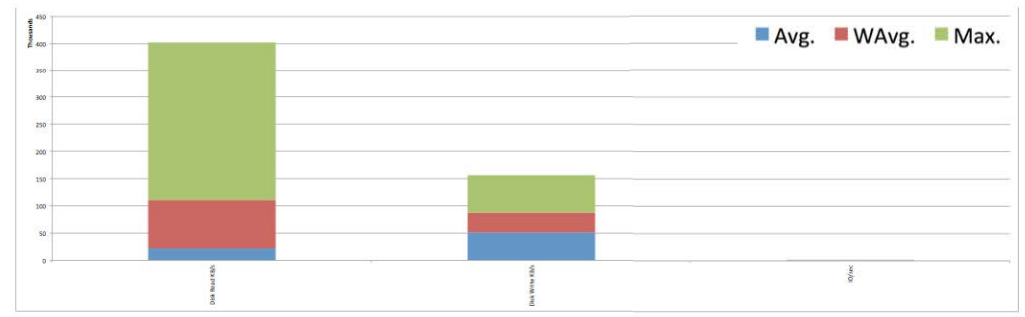

D

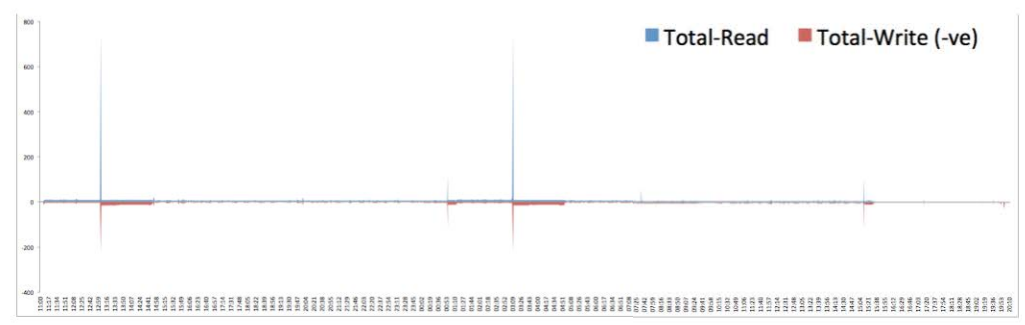

E

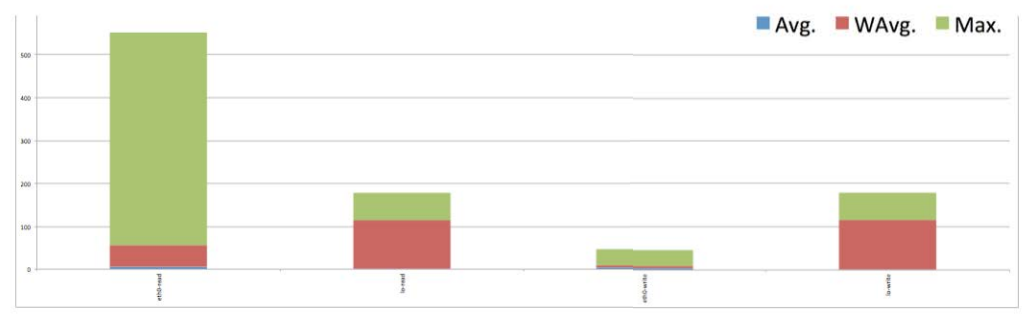

Figure 7.4: NMON Analyzer results for 1TB Terasort test with default parameters. (A) Total CPU Utilization, (B) CPU Utilization per CPU, (C) Disk I/O, (D) Network I/O, (E) Network Packets. 


\begin{tabular}{cccc}
\hline Test & Run & Total Time, sec & HSph@SF \\
\hline 1 & 1 & 50658 & 0.0710 \\
1 & 2 & 51541 & 0.0698 \\
2 & 1 & 56267 & 0.0639 \\
2 & 2 & 56306 & 0.0639
\end{tabular}

Table 7.2: TPCx-HS Default Baseline Tests Results

\begin{tabular}{ccc}
\hline Test & Total Time, ms & Total Time, min \\
\hline 1 & 5855681 & 98 \\
2 & 5528821 & 92 \\
3 & 5810725 & 97 \\
4 & 5897074 & 98 \\
5 & 5612621 & 94
\end{tabular}

Table 7.3: TeraSort on 100GB of data with Autotuner Empowered Hadoop with Hill Climbing Algorithm Tests Results

Network Packets activity are recorded on the master node $(\mathrm{D}, \mathrm{E})$.

\subsection{Preliminary Autotuner Results}

\subsubsection{TeraSort}

Testing of the Autotuner with the Hill Climbing algorithm was performed similarly to the default baseline: TeraSort, included in the Hadoop 2.6 examples package, was executed on $1 \mathrm{~TB}$ of data with default settings. The job was completed in 1 day 5 hours 49 minutes and 30 seconds.

Moreover, a series of 100 GB TeraSort tests was performed; completion times are presented in Table 7.3. 


\begin{tabular}{cccc}
\hline Test & Run & Total Time, sec & HSph@SF \\
\hline 1 & 1 & 62213 & 0.0578 \\
1 & 2 & 51077 & 0.0704 \\
2 & 1 & 55049 & 0.0653 \\
2 & 2 & 50935 & 0.0706
\end{tabular}

Table 7.4: TPCx-HS with Autotuner Empowered Hadoop with Hill Climbing Algorithm Tests Results

\subsubsection{TPCx-HS}

The TPCx-HS benchmark was executed on Hadoop empowered by Autotuner with the Hill Climbing algorithm, the configuration was left at default settings, identical to the default baseline setting described previously. Two TPCx-HS tests were performed, each test consists of two runs completion times and HSph@SF indicator for each of the four runs shown in Table 7.4.

\subsection{Explorer}

The Autotuner was tested with two Explorer Algorithms. First, explorer testing was performed with variable virtual core values and memory with the 30-second autotuner wave. Second, explorer testing was performed with the virtual cores value fixed at 1 and variable memory values with the 20-minute autotuner wave.

\subsubsection{Explorer with 30-second Autotuner waves on memory and vCores}

The explorer testing was performed with virtual core values 1, 2, 3 and 4 and memory values $640 \mathrm{MB}, 1024 \mathrm{MB}, 1224 \mathrm{MB}, 1920 \mathrm{MB}, 2560 \mathrm{MB}$, and $3200 \mathrm{MB}$ with the autotuner wave initiation length of 30 seconds. The average container completion times for autotuner waves, with respect to container memory and vCore settings, are shown in Figure 7.5. 


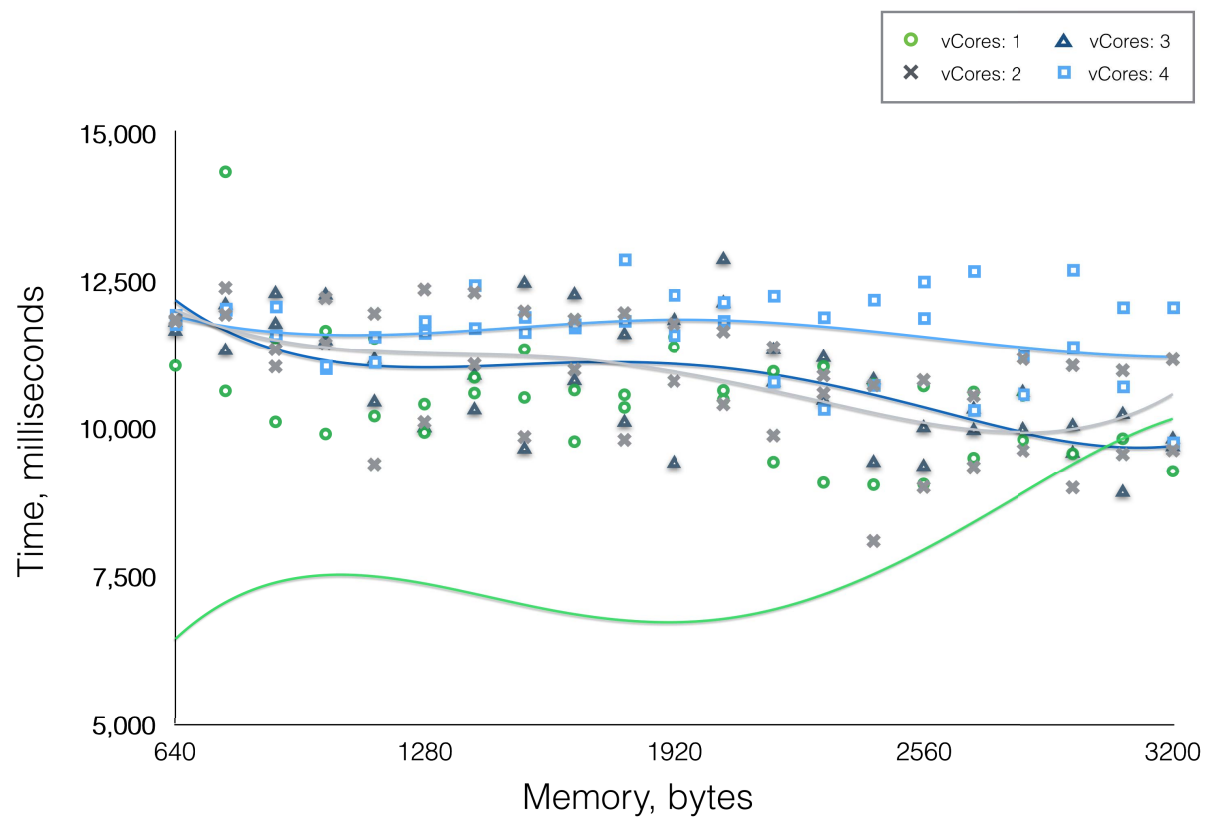

Figure 7.5: Hadoop empowered by the autotuner with Explorer algorithm with 30-second autotuner waves on memory and vCores

\subsubsection{Explorer with 20-minute Autotuner waves on memory}

The explorer testing was performed with the number of virtual cores fixed to 1 and variable memory with values of $640 \mathrm{MB}, 1024 \mathrm{MB}, 1224 \mathrm{MB}, 1920 \mathrm{MB}, 2560 \mathrm{MB}$ and 3200 MB. The autotuner wave initiation length was set to 20 minutes. The average container completion times for autotuner waves, with respect to container memory settings for the 1 TB TeraSort test, are shown in Figure 7.6. The average container completion times for the later $20 \%$ of autotuner waves with respect to container memory settings for the 1 TB TeraSort test are shown in Figure 7.7. The average container completion times for autotuner waves, with respect to container memory settings for the 2 TB TeraSort test, are shown in Figure 7.8. The average container completion times for the later $20 \%$ of autotuner waves, with respect to container memory settings for the 2 TB TeraSort test, are shown in Figure 7.9. 


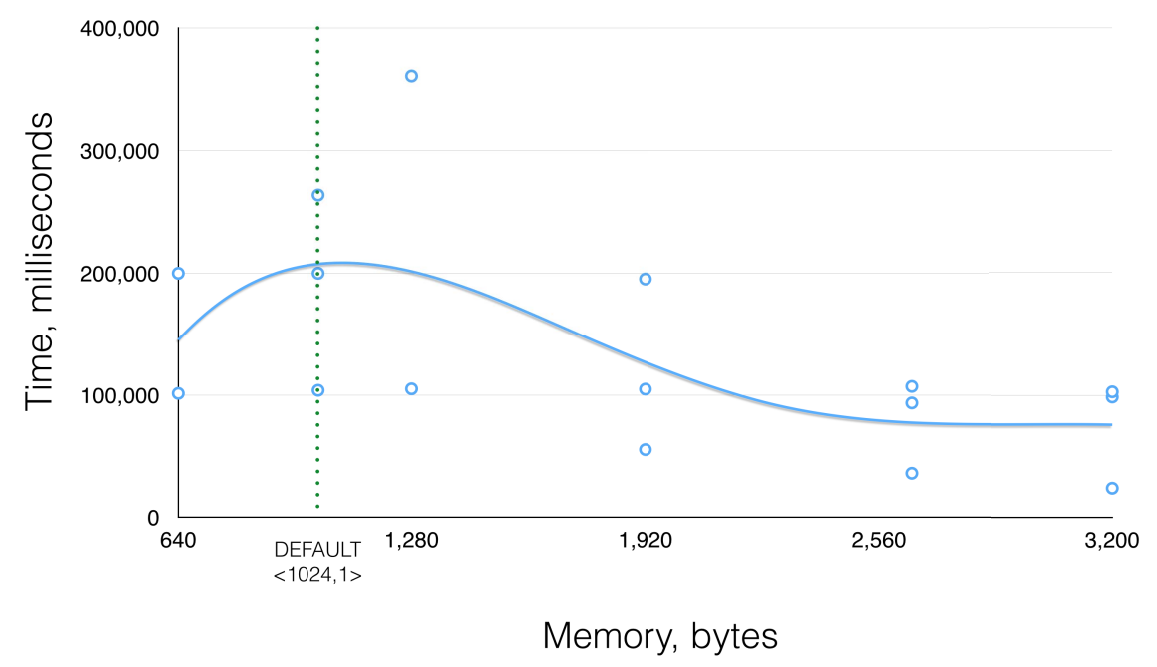

Figure 7.6: Hadoop empowered by the autotuner with Explorer algorithm with with 20-minute autotuner waves on memory for the 1 TB TeraSort

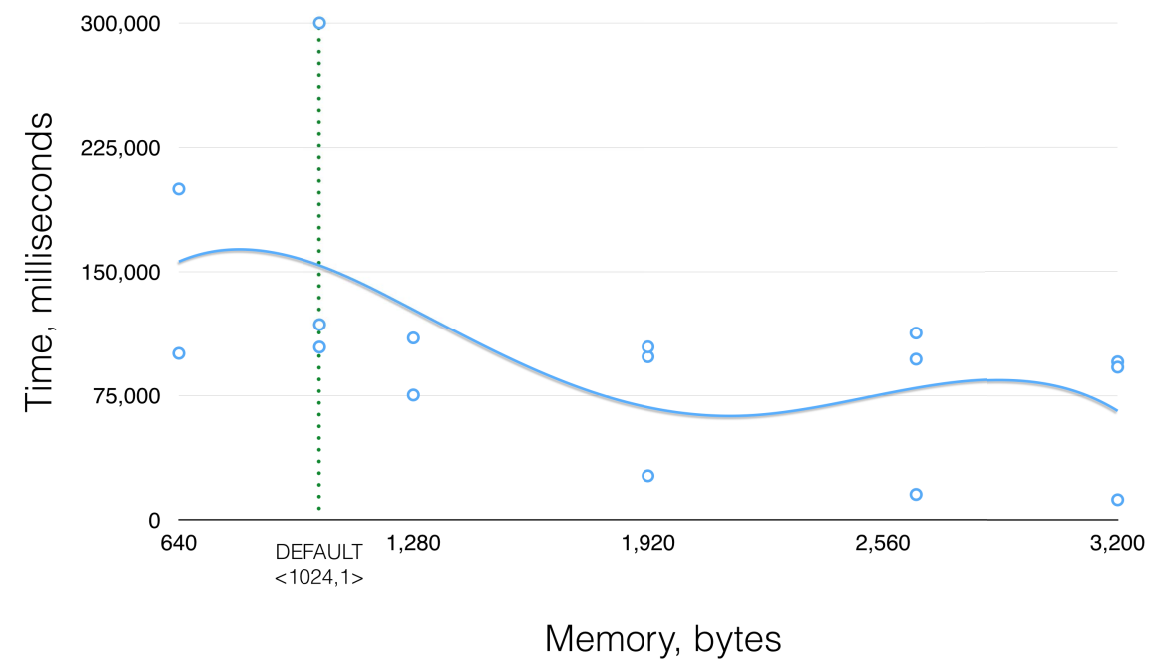

Figure 7.7: Hadoop empowered by the autotuner with Explorer algorithm with 20-minute autotuner waves on memory for the 1 TB TeraSort (the later 20\%) 


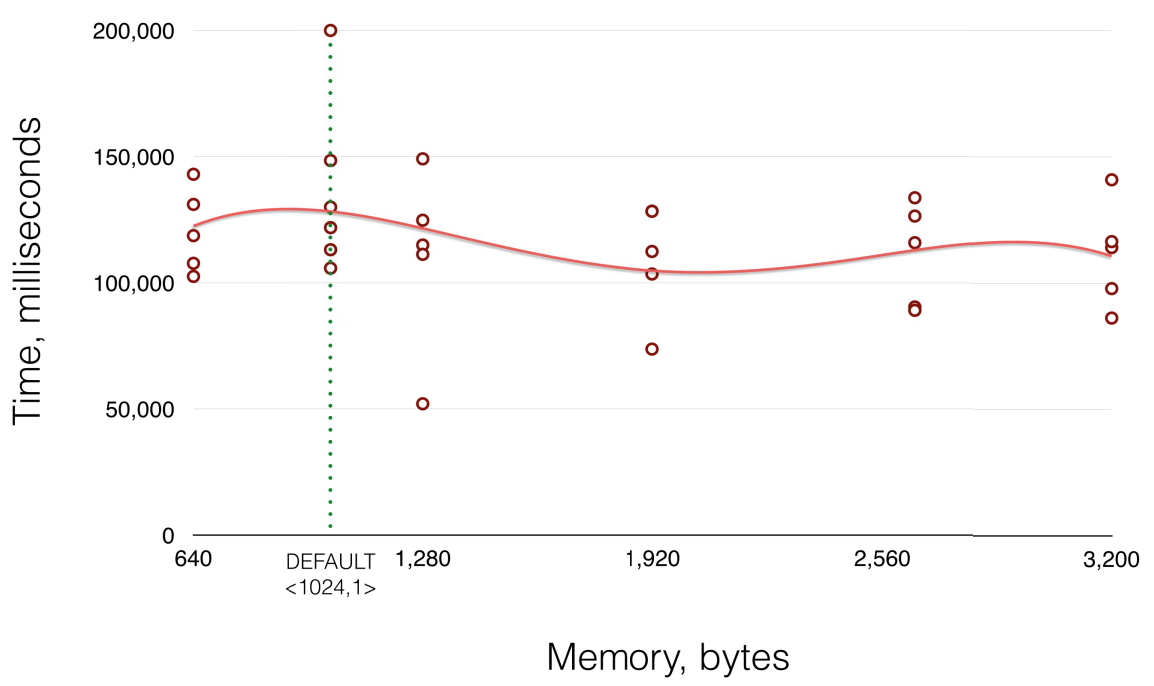

Figure 7.8: Hadoop empowered by the autotuner with Explorer algorithm with Explorer algorithm with 20-minute autotuner waves on memory for the 2 TB TeraSort

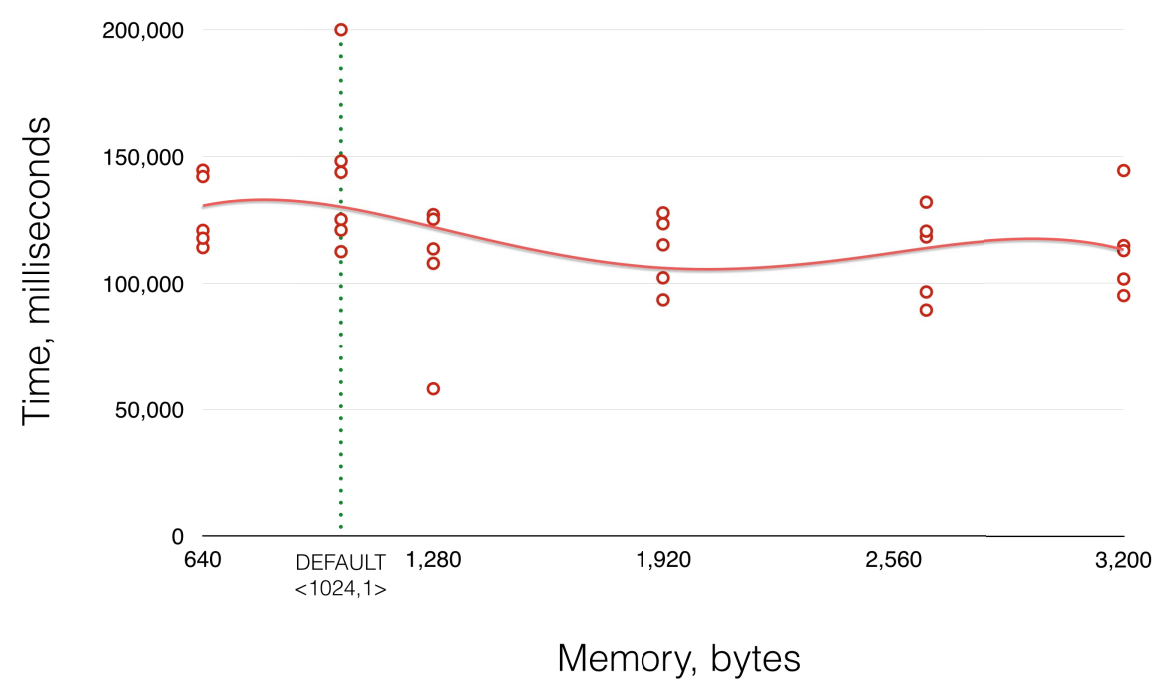

Figure 7.9: Hadoop empowered by the autotuner with Explorer algorithm with Explorer algorithm with 20-minute autotuner waves on memory for the $2 \mathrm{~TB}$ TeraSort (the later 20\%) 


\section{Chapter 8}

\section{Discussion}

\subsection{Innovation Concept. Real-Time Autotuning}

\subsubsection{Rational for real-time Hadoop YARN autotuning}

Hadoop exposes over 200 parameters, which drastically affect the cluster performance. Therefore, manual tuning has to be performed to achieve the desired results. Manual tuning is not only expensive, but also often unacceptable, because of the worldwide shortage of Hadoop tuning experts. Large companies, associated with Big Data technology, such as IBM, have teams of professionals dedicated to provide tuning services for the jobs run on Hadoop clusters.

Real-time autotuning is a highly desired, yet not explored problem in Hadoop YARN tuning. Considering typical use cases in business and academia, Hadoop is commonly deployed in situations in which the job is executed once or with a small number of repetitions. For instance, such jobs are common in the growing field of data science. In order to test the hypothesis on different sizes or the structure of data, optimal settings are different for every single execution must be tuned individually. Therefore, running the same job multiple times in order to obtain optimal parameter settings, even though it makes the single execution faster, overall, it makes it a few folds slower than the time achieved at default settings. Notice, that this will be the case in all off-line tuning algorithms, including the genetic algorithm, simulated annealing and hill climbing described earlier. For instance, the Gunther project employs a genetic algorithm, and produces optimal completion time under 12 generations. The obtained completion time was two times faster than original. Each of the generations must have taken at least optimal time. Therefore, to complete tuning, 
Gunther took 12 times the optimal job completion time or 6 times the worst (original) time. Hence, for the use cases in which a job is run under 6 times, the employment of Gunther could not be justified. All other off-line autotuners follow the same logic, which makes them not practical for the most popular Hadoop use cases. Therefore, real-time Hadoop YARN autotuning is a worthwhile and desirable research aim.

\subsubsection{Autotuner Design Requirements}

From the use cases mentioned earlier, desirable specifications for the autotuner architecture and functionality were derived. First and foremost, it was established that, for the most common Hadoop use cases, the total autotuning process should not take more than a single run on default settings. Therefore, an automatic tuning process must execute during a single Hadoop job.

In case of off-line autotuning, the cost function is based on information (such as completion time) obtained at the end of the job execution. Hence, the completion of the tuning within a time frame of a single default run is unfeasible. Therefore, a cost function must be developed, which estimates the total job completion time based on the information collected on a part of the job.

Furthermore, in order to on-line tune the parameters within the job execution, the autotuner must intercept tunables, collect cost evaluation, process information, produce new values for tunables and substitute old values with the new during execution on the Hadoop job. Therefore, the tuner must be tightly coupled with Hadoop YARN architecture, yet not interfere with the job flow. For instance, the autotuning process must be fast, in order to avoid lengthening the Hadoop job execution.

Moreover, it is desirable for the autotuner to be modular, with multiple algorithm options. Hence, a user can evaluate different tuning techniques employing the same real-time parameter changing mechanism.

\subsection{Design Decisions Motivation}

During the software design and implementation processes, a number of important design decisions were made. Let us review the most significant of the decisions in the current section. First, we will look at the rationale behind the selection of the tunables. Second, we will look at the point at which the Hadoop job flow must be 
accessed and modified. Third, we will look at the main modules of the autotuner and which tuning algorithms should be included in the initial prototype.

The selection of the tunables was based on two main factors: research of the published recommendations on Hadoop configuration tuning, and preliminary testing. Research of the potential tunables, which have sufficient impact on performance for MapReduce jobs on Hadoop/YARN architecture and applicable for real-time tuning was conducted. First, the full list of the parameters recommended to be tuned to optimize performance from various sources was compiled. Note that because of a recent transition to YARN-based architecture many previously important parameters became depreciated, or lost their effect on the system. Hence, the parameters were filtered accordingly. Next, a subset of parameters suitable for real-time tuning was selected and the most influential of the parameters were considered. Finally, a preliminary review of Hadoop/YARN architecture allowed us to select two sets of parameters, which were considered for the current research: mapreduce.*.memory.mb and mapreduce.*.cpu.vcores, where * stands for map and reduce. Commonly, map and reduce types of the parameters are set to the same value. Hence, for the current stage of the research, the map and reduce parameters were tuned to the same value. The selected parameters are specific to YARN-based Hadoop architecture and govern the size of memory and the number of virtual cores assigned to individual containers by the resource manager during the resource allocation process. Overall, the parameters allow indirect control of container granularity and density; therefore, they are affecting job completion time through the optimization of the cluster resource deployment.

In order to confirm that the parameters selected for the tuning have an impact and create non-trivial parameter space, a series of tests on a subset of parameter space was performed. A number of TeraSort jobs were executed with different mapreduce.map.memory.mb and mapreduce.reduce.memory.mb in configuration files. Completion times of the experiments are plotted against parameter values revealing multiple local minimum and maximum with default parameter been the global maximum (Figure 7.2 ). Such results suggest that the selected parameters are indeed the suitable subject for the tuning application.

The selected parameters are read from a mapred-site. $x m l$ configuration file and added to the job configuration object. Later, a job process uses it to calculate the complete set of containers required to complete the application. For MapReduce type 
jobs, containers are differentiated by the type of the task (map or reduce). Other types of jobs executed on the YARN base have homogeneous containers. In either case, the container configuration is passed to the resource manager via resource request. Each request is composed of the total number of containers needed to complete the job and amount of memory and virtual cores required per container. The Resource Request is updated and resent on every heartbeat. Two options were considered. The first option is to change the job configuration and create an additional mechanism, which would force an Application Master to recalculate the Resource Request based on the new set of container parameters. The second option is to interfere with already composed resource requests before their fulfillment, then change the values of the requested container memory and virtual cores. After careful consideration, which included Hadoop and YARN code investigation, understanding overall architecture intentions and dependencies, the second option was chosen. The modification of a Resource Request achieves the desired effects with the least amount of interference with the native Hadoop code without imposing an additional cycle. Resource Requests are sent repetitively based on the heartbeats, which orchestrate most of the processes accruing in Hadoop Yarn architecture. Therefore, employment of the heartbeat mechanism for the real-time autotuning processes is highly desirable.

Next, the decision was to select a place in a resource passing sequence for the autotuning interference. The interception could be done at an Application Master, before each request is sent; at the Resource Manager's Application Master Service, which governs container allocation calls validation; or at a YARN Scheduler, where a Resource Request is fulfilled. Note that a Resource Request is passed asynchronously between the Application Master and YARN site with the Resource Manager, which contains both an Application Master Service and Schedulers. Therefore, implementation on the YARN site allows the future extension of the Autotuner from the MapReduce to the wide range of other YARN-based applications. Moreover, YARN-based Resource Request interception is directly traceable and could be tested within the development environment on a Mini YARN Cluster. On the YARN site, both options, an Application Master Service and a Scheduler, were considered equally valid. Nevertheless, the main autotuner connection was chosen to be implemented on the base Application Master Service class as the autotuner purpose is in tighter agreement with the main roles of the Application Master Service class in the YARN architecture.

Even though the autotuner was built with modularity as the key design principle 


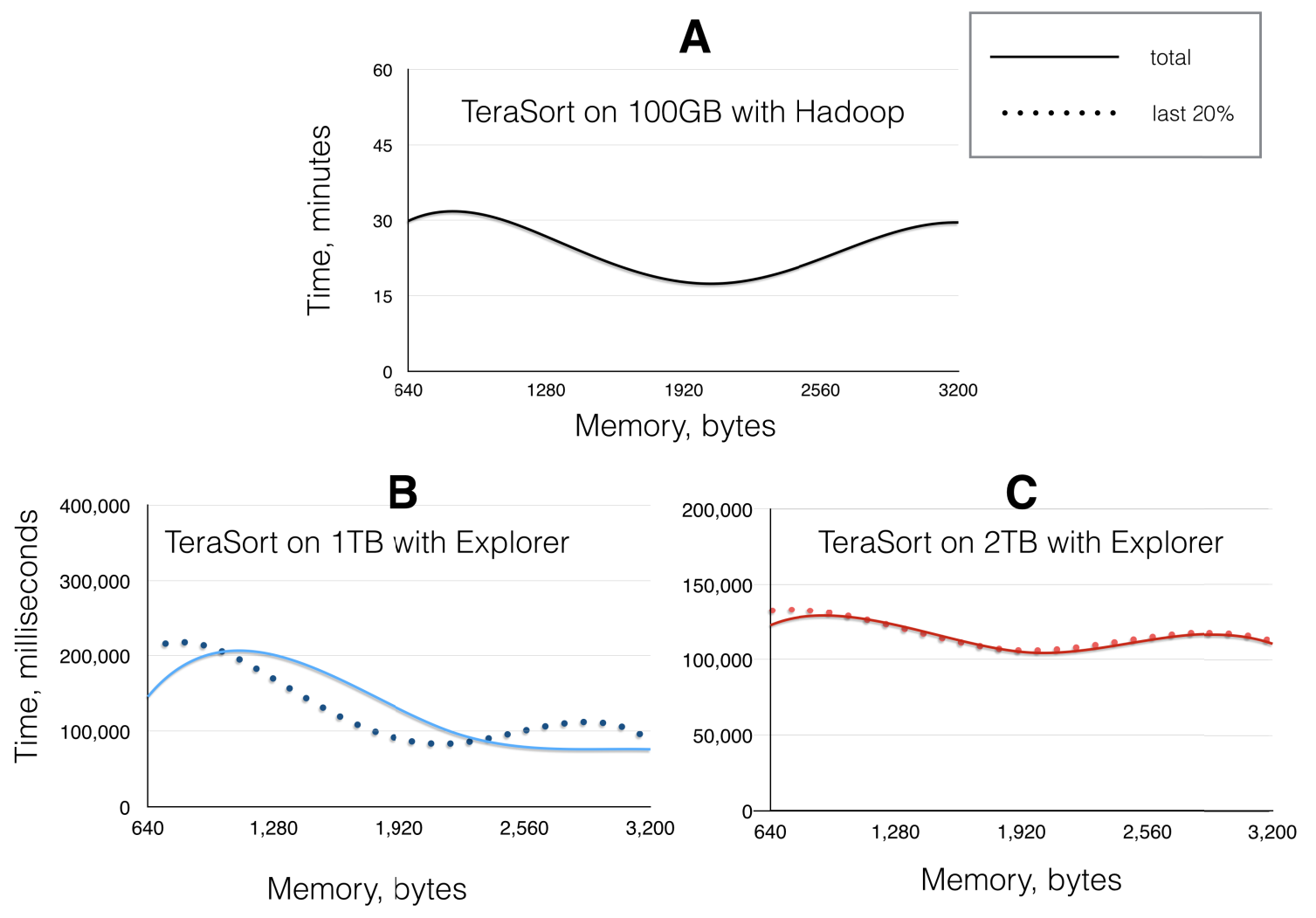

Figure 8.1: Comparison of the trend-lines: (A) Complete Terasort execution times, (B) Average container completion time per the autotuner wave during 1TB Terasort, (C) Average container completion time per the autotuner wave during 2TB Terasort

and, in future selection among multiple algorithms, is extremely desirable at this stage, one algorithm must be selected to limit the scope of the current project to a manageable size. A number of popular algorithms used for non-unimodal parameter space tuning were considered including genetic, simulated annealing and hill climbing algorithms. A hill climbing algorithm was chosen for its simplicity and absence of additional parameters, which would create additional tunables for the user. 


\subsection{Result Evaluation}

\subsubsection{Comparison of the Preliminary Autotuner Runs with Default and RoT Testings}

The comparison of the trend-lines was produced by plotting multiple complete Hadoop Terasort executions completion times against the memory parameter value (Figure $8.1(\mathrm{~A})$ ) and trend-lines produced by the average container completion time per the autotuner wave against container memory size value for the wave for Terasort Executions on $1 \mathrm{~TB}$ and $2 \mathrm{~TB}$ of data (Figure 8.1 (B),(C)). Note Figure 8.1 combines Figures 7.2, 7.6, 7.7, 7.8, and 7.9 in order to expose striking similarity in trend-line shapes. The difference in scales between complete job executions and the average wave container completion times prevents from joining all graphs in one. Nevertheless, analogous behaviour of all trend-lines is unquestionable. Moreover, the exclusion of first $80 \%$ of containers, in order to illuminate possibility of the effects carried from the previous waves. The graph comparison shows that the illumination of the earlier containers positively effects the $1 \mathrm{~TB}$ execution, yet has no effect on the 2 TB Terasort execution trend-lines. Hence, scheduling mechanisms, in some cases, prevent clear separation among the waves and the previous wave could affect completion of the first containers of the wave. This could be addressed by providing an autotuner-specific YARN Scheduler, which could provide better wave segregation.

\subsubsection{Explanation of the Results Obtained by Preliminary Autotuner Testing and Explorer Execution}

One of the valid explanations for the absence of the improvement is a problem with the implementation mechanism or with cost function. For example, the additional time the autotuner takes to calculate a new set of parameters and pass them to the Application Master Service is excessive. In order to test the hypothesis, time stamps were taken before and after the autotuner is called to tune. The result shows on average $3 \mathrm{~ms}$ per the autotuner wave. Hence, the time that autotuner adds to the overall execution is marginal and cannot explain the behaviour.

The next hypothesis is that the altering Resource Request is not a sufficient mechanism to alter the container size during job execution. Moreover, the cost function 
is based on the average container completion time of the wave, which could be ineffective. In order to validate the two hypothesis, an explorer test was performed. First, with 30-second and 1-minute lengths of the wave, both show high variation in container completion times within the waves set to the same memory and the number of virtual cores per container. The results shown in Figure 8.1 did not exhibit a correlation between variation in full job run completion times and average container completion time; yet, the result cannot be considered final as multiple additional reasons could be playing a part in the outcome. For example, waves are not long enough to clear the queue; hence, containers from previous waves temporally overlap, the current wave containers creating excessive noise in the data. From this observation, an idea to elongate wave lengths was derived. Moreover, data have shown that, in all testings, the number of the virtual cores directly correlates with container completion time. Therefore, the best time is always exhibited by the vCores parameter set to one. In order to obtain more readable results, new explorer testing was performed with the vCores parameter fixed to a single core and a wave length of 20 minutes. Obtained results for Terasort on 1TB and 2TB of data were plotted as is shown in Figure 7.6,7.8 and directly correspond to the trend line obtained for the full job executions, which is displayed in Figure 8.1. This result not only strongly supports the cost function, but also confirms the effectiveness of the autotuner implementation mechanism, as the resulting autotuner wave time directly correlates with the full job completion time.

Moreover, the observed variation in average container completion times could serve as a sufficient explanation of autotuner inefficiency in the current state. A hill climbing algorithm has very little chance to work towards improvement given the size of the improvement and the range of the fluctuation among the values for the same parameter settings. As it clearly shows in Figure 7.2, starting at the default value, even though, on average, default is the worst performing setting, there is a great chance that the hill climbing algorithm will not find a better neighbour and sets there. Hence, complete execution will be performed with the default settings. Therefore, in order to achieve effective improvement in completion times, the level of noise in the completion times must be lowered. 


\subsubsection{Strength of the Current Autotuner Design}

As a result of this work, the autotuner implementation was developed, which not only works, but also works efficiently. Considering how short the time that autotuner takes in comparison to the total job time, it practically does not impose any additional cost to run.

Moreover, it includes an accurate cost function, which correctly estimates the complete job running time based on a test (autotuner wave) performed on a small portion of the job. The accuracy of the evaluation confirmed with explorer tests is summarized in Figure 8.1.

Moreover, the Autotuner was placed strategically among the YARN architecture and localized within the Resource Manager package. Hence, deployment of the Autotuner is extremely simple even on an existent Hadoop cluster.

The modularity of the Autotuner design allows for easy extensions with additional algorithms via a simple inheritance mechanism. For example, multiple algorithm classes could be implemented as extensions of Algo. Moreover, additional parameters could be tuned and could simply be added to the Parameters object.

Furthermore, the steps towards the understanding of observed variation among the data points was taken. A group of hypotheses was considered. First, such variation could be triggered by a wave influences extended to the next wave for some period of time. In particular, it reflects how the scheduler propagates the Resource Request configuration to the actual resource allocation. As a scheduler does not implement changes unless currently running, containers complete their tasks and a scheduler queue is repopulated with containers with new memory and virtual core number settings. Hence, in order to test this hypothesis, Explorer test data was filtered, which excluded containers influenced by a previous wave (only last $20 \%$ of the container were taken into account). Such a measure must exclude containers influenced by a previous wave. Results show that only the smaller job (Terasort on 1TB of data) with less data points produces a slightly improved trend line, which more closely corresponds to the complete job execution parameter exploration graph (see Figures 7.7). Nevertheless, the larger job (2TB Terasort) did not exhibit significant changes in trend line behaviour. Moreover, graphs created by complete container data and by the last $20 \%$ of the containers are almost identical (see Figures 7.9). Hence, the autotuning mechanism is not significantly influenced by the temporal overlapping between waves created by the scheduler implementation, especially for larger workloads. 


\subsubsection{Weakness of the Current Autotuner Design and Ideas for Improvement}

Currently no significant improvement of the map reduce runs on Hadoop 2.2 with YARN was observed. As was previously discussed, a number of factors could be the reason, or a part of the reason, for such a result. It was determined that the parameters selected for tuning have an impact on the job run time and that the mechanism for parameter changing in real-time is effectively changes parameters and receives corresponding evaluation. Hence, the inefficiency of the autotuner must take root in the tuning algorithm and the specifics of the data. In particular, observed high variation for each memory setting, especially in relation with achievable improvement, creates a low probability for a hill climbing algorithm to improve the current state.

Hence, improvement could be achieved by gaining insight on causes of the variation and lowering its levels. The possible reasons for such behaviour could be divided in two classes: software related and hardware related. Hardware related variation is based on the fact that testing cluster is composed of only four nodes, and only three of them carry actual computation as data nodes. Therefore, any inconsistencies in performance, partial or complete failures have a considerable impact on performance, despite the renowned Hadoop robustness.

Next, software related reasons caused by the excessive variance should be considered. Hadoop and YARN compose complex software framework, which could conceal multiple reasons for such behaviour. For example, taking into account the slight trend line improvement for a smaller job that exclusion of the first $80 \%$ of container completion times weighted average scheme or more custom tailored for the work with the Autotuner YARN scheduler could be considered. Such implementation can reduce variance among the waves.

In addition, the variation between container completion times could be triggered by the heterogeneity of the tasks completed by individual containers. In particular, a few individual containers of the MapReduce job seem to be running up to ten fold longer than an average container. Moreover, map and reduce tasks could require different settings. Therefore, the autotuner could benefit from the delayed start and two

mode tuning, which differentiates between containers produced for map and reduce tasks.

Moreover, extending rules of thumb selection to a wider range of parameter may help stabilize container completion time variation. Nevertheless, similar variations in 
the total job completion times for the original Hadoop installation strongly suggest that roots of the observed noise lay outside of the autotuner, and must be addressed on the cluster level. 


\section{Chapter 9}

\section{Summary}

\subsection{Overview of the Results}

Current work emphasizes the importance of real-time automatic tuning method and proves the existence of a mechanism for utilization of the idea on the current Hadoop/YARN architecture.

A novel real-time autotuning approach for MapReduce jobs executed on Hadoop/YARN framework was proposed and implemented. The autotuner implementation connected to Hadoop/YARN through the Resource Manager and was built in a modular, extendable manner. The parameters, responsible for configuration of individual containers, were tuned between execution waves by the autotuner. The effectiveness of the autotuner's method of Resource Request interception and modification was experimentally proven. Moreover, a valid cost evaluating function was developed.

Furthermore, initial autotuner test results were evaluated and steps toward disclosure of the issues preventing the autotuner from fulfillment of its objectives were made. Moreover, a road map of further development for the autotuner composed of prioritized items in the desired order was composed. 


\subsection{Future Development}

The developed autotuner is the basis for a much larger project. The road map of the future development could be divided in a number of steps. Such steps form an agenda of goals, which should be executed in order. All steps could be divided in three groups based on priority.

The first group of the future development is the most critical. It includes steps that will bring the autotuner to the minimal pragmatically compelling state. First, the source of the noise must be found. A number of hypotheses should be tested. For instance, if noise is a result of the small cluster size and hardware state, then a test on a larger cluster will show more stable results. Another reason for the noise could be the dual nature of a MapReduce job. Containers with the same parameters could not only run map and reduce tasks with different speeds, but also react differently on parameter changes. For example, an increase in memory at some point could speedup map containers, but slow down reduce tasks, creating the noise. To test this hypothesis, the job composed from map or reduce tasks only should be run, such as TeraGen, for example.

Next, when the source of variation is found, noise should be reduced to the level accepted by the tuning algorithms. This step will depend on the source nature. For example, in a case of hardware dependence, recommendations for a minimal size of a cluster, on which the autotuner should be employed, could be made. In case of map and reduce task differences, causation for the noise, implementation of two phase tuning mechanisms and individual for each of a task type could be considered.

The second group of future developments expands the autotuner project. A number of autotuning algorithms could be increased. Moreover, an existing hill climbing algorithm could be improved using successful techniques such as the Late Acceptance Hill Climbing algorithm. Next, an autotuner-specific scheduler could be built, in order to propagate tuning to another level. Moreover, a number of parameters selected for real-time, automatic tuning could be increased. Another way of improving the autotuner could be increased, coupling real-time automatic tuning with a system of rule-based tuning, which converts out of the box default configuration to the one closer to a rule-of-thumb tuned state before a job execution process. Cost evaluating function should take into account not only container completion time, but also the overall structure of the cluster resources employment in order to minimize idle state.

The third group of future development addresses the deployment process. It 
includes an addition of a simple user interface and a parameterization of some of the currently fixed values, such as algorithm selection, or the autotuner wave length. Creation of Hadoop patch. Moreover, making the autotuner available for installation on top of the current Apache Hadoop should be set as the final goal of the project.

Encapsulating the significance of the work undertaken within this project the extensive scope and enormous practical value of the final goal must be acknowledged. This project laid a strong base for farther advancement towards the goal. 


\section{List of References}

[1] T. O. E. Dictionary, "Big data."

[2] D. Laney, "3d data management: controlling data volume, velocity and variety. meta group, ht tp," blogs. gartner. com/doug-laney/files/2012/01/ad949-3DData-Management-Controlling-Data-Volume-Velocity-and-Variety. pdf (accessed 16 January 2013), 2001.

[3] T. H. Davenport and D. Patil, "Data scientist: The sexiest job of the 21st century-a new breed of professional holds the key to capitalizing on big data opportunities. but these specialists aren't easy to findand the competition for them is fierce.," Harvard Business Review, p. 70, 2012.

[4] J. Dean and S. Ghemawat, "Map-reduce: Simplified data processing on large clusters," D OSDI IEEE, 2004.

[5] J. Dean and S. Ghemawat, "Mapreduce: simplified data processing on large clusters," Communications of the ACM, vol. 51, no. 1, pp. 107-113, 2003.

[6] S. Ghemawat, H. Gobioff, and S.-T. Leung, "The google file system," in ACM SIGOPS operating systems review, vol. 37, pp. 29-43, ACM, 2003.

[7] I. D. Corporation, "Idc releases first worldwide hadoop-mapreduce ecosystem software forecast, strong growth will continue to accelerate as talent and tools develop," 2012.

[8] H. Herodotou, H. Lim, G. Luo, N. Borisov, L. Dong, F. B. Cetin, and S. Babu, "Starfish: A self-tuning system for big data analytics.," in CIDR, vol. 11, pp. 261-272, 2011.

[9] H. Herodotou and S. Babu, "Profiling, what-if analysis, and cost-based optimization of mapreduce programs," Proc. of the VLDB Endowment, vol. 4, no. 11, pp. 1111-1122, 2011.

[10] G. Liao, K. Datta, and T. L. Willke, "Gunther: search-based auto-tuning of mapreduce," in Euro-Par 2013 Parallel Processing, pp. 406-419, Springer, 2013.

[11] Y. Chen, M. Winslett, Y. Cho, and S. Kuo, "Automatic parallel i/o performance optimization in panda," in Proceedings of the tenth annual ACM symposium on Parallel algorithms and architectures, pp. 108-118, ACM, 1998. 
[12] A. Bialecki, M. Cafarella, D. Cutting, and O. OMalley, "Hadoop: a framework for running applications on large clusters built of commodity hardware, 2005," Wiki at http://lucene. apache. org/hadoop, 2005.

[13] M. Zaharia, M. Chowdhury, M. J. Franklin, S. Shenker, and I. Stoica, "Spark: cluster computing with working sets," in Proceedings of the 2nd USENIX conference on Hot topics in cloud computing, vol. 10, p. 10, 2010.

[14] P. Mundkur, V. Tuulos, and J. Flatow, "Disco: a computing platform for largescale data analytics," in Proceedings of the 10th ACM SIGPLAN workshop on Erlang, pp. 84-89, ACM, 2011.

[15] S. Plimpton and K. Devine, "Mapreduce-mpi library," Sandia National Laboratories. Available fr om http://www. sandia. gov/ ${ }^{\sim}$ sjplimp/mapreduce. html, accessed on March, vol. 4, 2013.

[16] E. Dede, Z. Fadika, J. Hartog, M. Govindaraju, L. Ramakrishnan, D. Gunter, and R. Canon, "Marissa: Mapreduce implementation for streaming science applications," in E-Science (e-Science), 2012 IEEE 8th International Conference on, pp. 1-8, IEEE, 2012.

[17] R. M. Yoo, A. Romano, and C. Kozyrakis, "Phoenix rebirth: Scalable mapreduce on a large-scale shared-memory system," in Workload Characterization, 2009. IISWC 2009. IEEE International Symposium on, pp. 198-207, IEEE, 2009.

[18] A. S. Foundation, "Determine yarn and mapreduce memory configuration settings," 2014.

[19] A. S. Foundation, "Determine yarn and mapreduce memory configuration settings," 2014.

[20] C. A. Schaefer, V. Pankratius, and W. F. Tichy, "Atune-il: An instrumentation language for auto-tuning parallel applications," in Euro-Par 2009 Parallel Processing, pp. 9-20, Springer, 2009.

[21] M. J. Cafarella and C. Ré, "Manimal: relational optimization for data-intensive programs," in Procceedings of the 13th International Workshop on the Web and Databases, p. 10, ACM, 2010.

[22] N. Yigitbasi, T. L. Willke, G. Liao, and D. Epema, "Towards machine learningbased auto-tuning of mapreduce," in Modeling, Analysis \&3 Simulation of Computer and Telecommunication Systems (MASCOTS), 2013 IEEE 21st International Symposium on, pp. 11-20, IEEE, 2013.

[23] T. Lipcon, "Cloudera: 7 tips for improving mapreduce performance," 2009.

[24] T. Lipcon, "Cloudera: Optimizing mapreduce job performance," 2012.

[25] Impetus, "Hadoop performance tuning," 2012. 
[26] B. Behzad, S. Byna, S. M. Wild, M. Prabhat, and M. Snir, "Improving parallel i/o autotuning with performance modeling," in Proceedings of the 23rd International Symposium on High-performance Parallel and Distributed Computing, HPDC '14, (New York, NY, USA), pp. 253-256, ACM, 2014.

[27] J. Liu, N. Ravi, S. Chakradhar, and M. Kandemir, "Panacea: towards holistic optimization of mapreduce applications," in Proceedings of the Tenth International Symposium on Code Generation and Optimization, pp. 33-43, ACM, 2012.

[28] Q. Chen, M. Guo, Q. Deng, L. Zheng, S. Guo, and Y. Shen, "Hat: historybased auto-tuning mapreduce in heterogeneous environments," The Journal of Supercomputing, vol. 64, no. 3, pp. 1038-1054, 2013.

[29] E. R. Lucas Filho, E. C. De Almeida, Y. Le Traon, et al., "Intra-query adaptivity for mapreduce query processing systems," in IDEAS 2014: 18th International Database Engineering 85 Applications Symposium, 2014.

[30] M. Li, L. Zeng, S. Meng, J. Tan, L. Zhang, A. R. Butt, and N. Fuller, "Mronline: Mapreduce online performance tuning," in Proceedings of the 23rd international symposium on High-performance parallel and distributed computing, pp. 165-176, ACM, 2014.

[31] S. Kirkpatrick, C. D. Gelatt, M. P. Vecchi, et al., "Optimization by simulated annealing," science, vol. 220, no. 4598, pp. 671-680, 1983.

[32] V. Černỳ, "Thermodynamical approach to the traveling salesman problem: An efficient simulation algorithm," Journal of optimization theory and applications, vol. 45, no. 1, pp. 41-51, 1985.

[33] A. M. Turing, "Computing machinery and intelligence," Mind, pp. 433-460, 1950

[34] N. A. Barricelli, "On the manner in which crossbreeding takes place in bacteriophages and bacteria," Acta Biotheoretica, vol. 11, no. 2, pp. 75-84, 1955.

[35] B. Behzad, H. V. T. Luu, J. Huchette, S. Byna, R. Aydt, Q. Koziol, M. Snir, et al., "Taming parallel i/o complexity with auto-tuning," in Proceedings of the International Conference on High Performance Computing, Networking, Storage and Analysis, p. 68, ACM, 2013.

[36] B. Selman and H. Kautz, "Domain-independent extensions to gsat: Solving large structured satisfiability problems," in IJCAI, vol. 93, pp. 290-295, Citeseer, 1993.

[37] E. K. Burke and Y. Bykov, "The late acceptance hill-climbing heuristic," University of Stirling, Tech. Rep, 2012.

[38] A. S. Foundation, "Hadoop mapreduce next generation - cluster setup," 2014.

[39] Hortonworks, "Determine yarn and mapreduce memory configuration settings," 2014.

[40] IBM, "nmon," 2013. 


\section{Appendix A}

\section{The List of Imports for a MiniYARNCluster}

- java.io.IOException;

- java.util.StringTokenizer;

- org.apache.hadoop.conf.Configuration;

- org.apache.hadoop.fs.Path;

- org.apache.hadoop.io.IntWritable;

- org.apache.hadoop.io.Text;

- org.apache.hadoop.mapreduce.Job;

- org.apache.hadoop.mapreduce.Mapper;

- org.apache.hadoop.mapreduce.Reducer;

- org.apache.hadoop.mapreduce.lib.input.FileInputFormat;

- org.apache.hadoop.mapreduce.lib.output.FileOutputFormat;

- org.apache.hadoop.util.GenericOptionsParser;

- org.apache.hadoop.yarn.conf.YarnConfiguration;

- org.apache.hadoop.yarn.server.MiniYARNCluster;

- org.apache.hadoop.yarn.server.resourcemanager.scheduler.ResourceScheduler; 
- org.apache.hadoop.yarn.server.resourcemanager.scheduler.fifo.FifoScheduler;

- java.io.File;

- java.io.FileOutputStream;

- java.io.OutputStream;

- java.net.URL;

- org.apache.commons.logging.Log;

- org.apache.commons.logging.LogFactory; 